\title{
General Approach toward Aspidospermatan-Type Alkaloids Using One-Pot Vilsmeier-Haack Cyclization and Azomethine Ylide Cycloaddition
}

\author{
Clémence Hauduc and Guillaume Bélanger*10
}

Département de Chimie, Université de Sherbrooke 2500 boulevard Université, Sherbrooke, Québec J1K 2R1, Canada

Supporting Information

ABSTRACT: The development of a new one-pot reaction sequence afforded the tricyclic core of several aspidospermatan-type alkaloids from a linear, densely functionalized substrate. The key sequence features a highly chemoselective formamide activation that triggered a Vilsmeier-Haack cyclization, followed by an azomethine ylide generation and intramolecular cycloaddition. The choice of nucleophile, azomethine ylide precursor, and dipolarophile was crucial to the success of the sequence.

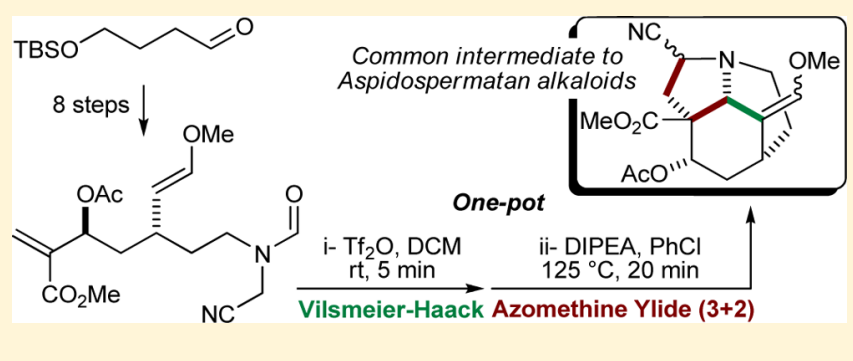

Scheme 1. Retrosynthesis Analysis of Common Precursor 4
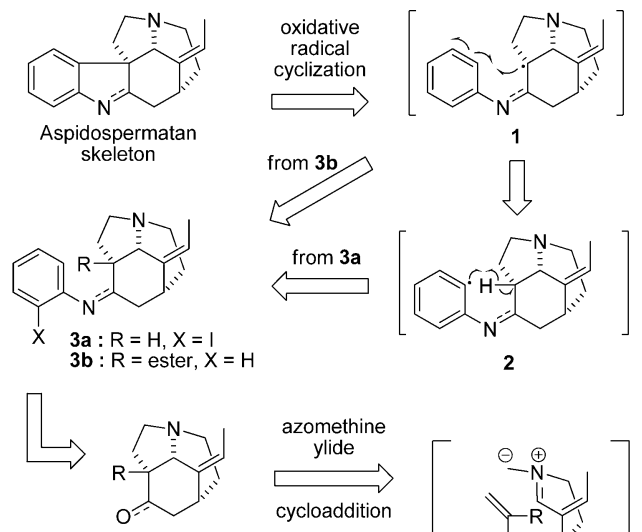

$$
\mathrm{O}
$$

Common precursor 4 $(\mathrm{R}=\mathrm{H}$ or ester)

As part of our research in the development of new one-pot reaction sequences to access functionalized cores of polycyclic alkaloids, ${ }^{2}$ we envisaged a unified approach toward several members of the aspidospermatan-type alkaloids. Key intermediate $\mathbf{4}$ was seen as a common precursor to a series of natural products by functional group manipulations and introduction of the indoline moiety using the ketone on 4 (Scheme 1$){ }^{3}$ After amination, the indoline would be prepared by radical dehalogenation of $\mathbf{3 a}$, followed by hydrogen abstraction on intermediate 2 and oxidative radical cyclization. ${ }^{4}$ Alternatively, the same intermediate $\mathbf{1}$ may also be accessed from a radical decarboxylation of ester $\mathbf{3 b}$. $^{5}$ Common precursor $\mathbf{4}$ would be the direct product of the highlighted one-pot Vilsmeier-Haack cyclization of formamide $\mathbf{6}$ upon activation and nucleophilic trapping, followed by in situ azomethine ylide 5 generation and intramolecular cycloaddition. The required amide 6 would be derived from nitro 7, the enone (dipolarophile) from an alcohol, and the nucleophilic allylsilane from aldehyde 7 . The latter would come from alkylation of protected hydroxyaldehyde $\mathbf{8}$.

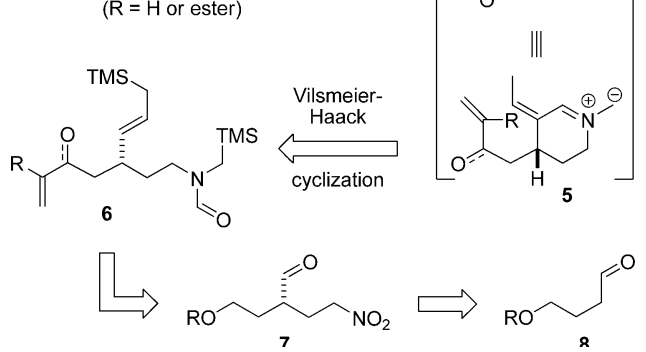

\section{RESULTS AND DISCUSSION}

The synthesis of the key step substrate 6 started with known aldehyde $9^{6}$ that was subjected to a pyrrolidine catalyzed conjugate addition to nitroethylene (Scheme 2). ${ }^{7}$ The allylsilane

Received: February 13, 2017

Published: April 18, 2017 
Scheme 2. Synthesis of Key Step Substrate 6
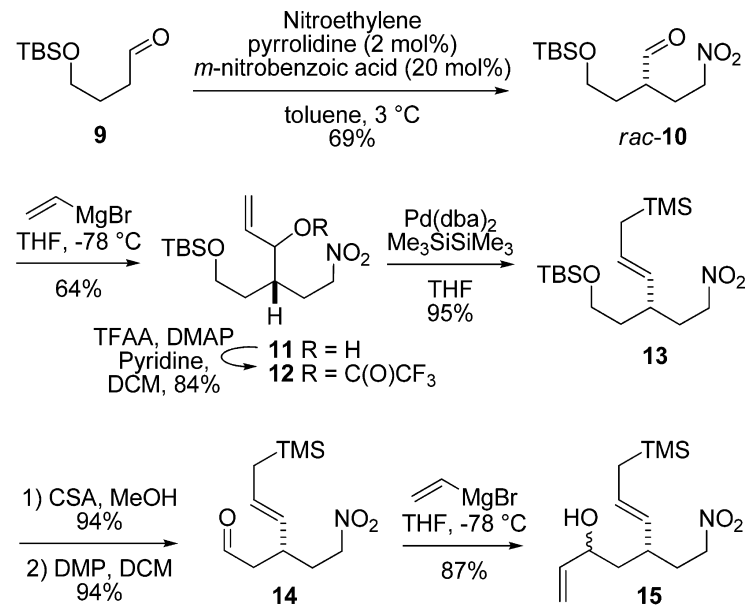
AcOEt 2) $\mathrm{ICH}_{2} \mathrm{TMS}, \mathrm{K}_{2} \mathrm{CO}_{3}$ THF, Reflux

3) $\mathrm{N}(\mathrm{CHO}) \mathrm{Bt}, \mathrm{THF}$

$63 \%$ (3 steps)

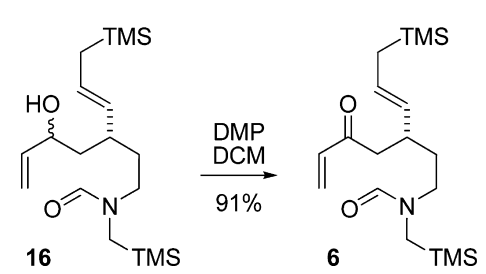

nucleophile required for the Vilsmeier-Haack reaction was installed on racemic aldehyde $\mathbf{1 0}$ through a vinyl Grignard addition, acylation, and $\mathrm{S}_{\mathrm{N}} 2^{\prime}$ displacement with TMS using Tsuji's conditions. ${ }^{8}$ Acidic methanolysis of silyl ether $\mathbf{1 3}$ followed by Dess-Martin oxidation produced aldehyde 14. After another vinyl Grignard addition, the resulting allylic alcohol 15 was obtained as a 1:1 mixture of diastereomers. ${ }^{9}$ The nitro group was then reduced with zinc dust in weak acidic conditions ${ }^{10}$ that were tolerated by the sensitive allylsilane. The resulting amine was alkylated with (iodomethyl)trimethylsilane and then formylated using Katritzky's reagent ${ }^{11}$ to afford formamide 16. A DessMartin periodinane (DMP) oxidation of the latter generated compound 6 ready for testing the full cyclization sequence.

Key step substrate 6 was treated with triflic anhydride in the presence of 2,6-di-tert-butyl-4-methylpyridine (DTBMP) to effect the amide activation and Vilsmeier-Haack reaction (Scheme 3). Unfortunately, we were not able to observe the formation of iminium ion $\mathbf{1 7}$ or $\mathbf{1 8}$ by ${ }^{1} \mathrm{H}$ NMR spectral analysis, although following such reactions using this technique is routinely done in our laboratory. ${ }^{2}$

At this point, we hypothesized that the enone was too reactive and did not withstand the activation conditions, even when the reaction was started at $-78{ }^{\circ} \mathrm{C}$. To confirm this hypothesis,

Scheme 3. Attempted Vilsmeier-Haack/Azomethine Ylide Cycloaddition Sequence with Enone 6
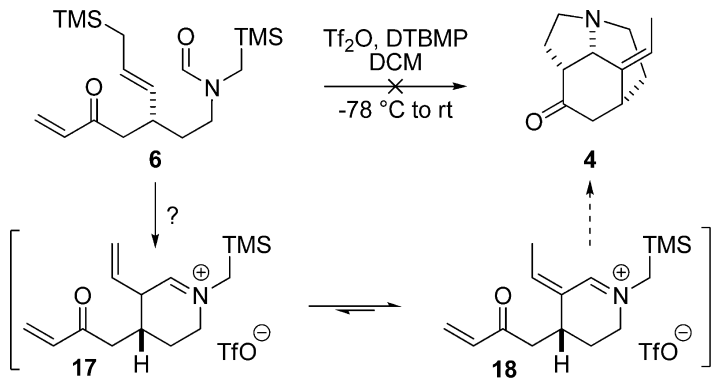

substrate 19 was prepared by benzylation of alcohol 16 (Scheme 4). When formamide 19 was activated, nonconjugated iminium

Scheme 4. Attempted Vilsmeier-Haack/Azomethine Ylide Cycloaddition Sequence with Protected Allylic Alcohol 19
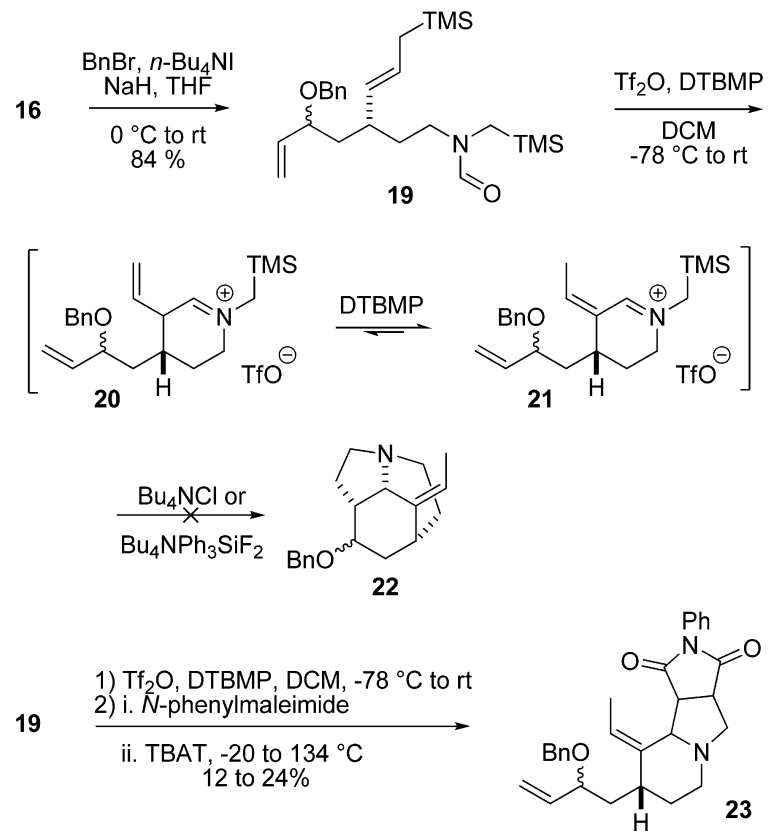

ion intermediate 20 was observed by ${ }^{1} \mathrm{H}$ NMR spectroscopy. After $4 \mathrm{~h}$ at room temperature, conversion into eniminium ion $\mathbf{2 1}$ was complete. Conjugation could also be effected by addition of an excess of DTBMP, or by purification of the iminium ion intermediate using flash chromatography. Unfortunately, any attempt to generate the azomethine ylide by desilylation of $\mathbf{2 0}$ or 21 and further intramolecular trapping of the corresponding azomethine ylide failed.

This result did not allow a conclusion as to whether the failure to generate cycloadduct $\mathbf{2 2}$ was due to a reluctant desilylation of iminium ion $20 / 21$ or to a lack of reactivity of either the corresponding azomethine ylide or the dipolarophile in the cycloaddition step. Because this information was crucial to design a successful cycloaddition, we purified iminium ion 21 and treated the latter with tetrabutylammonium difluorotriphenylsilicate (TBAT) in the presence of an excess of $\mathrm{N}$-phenylmaleimide at different temperatures. Cycloadduct $\mathbf{2 3}$ was obtained, albeit in moderate yield. ${ }^{12}$ Hence, these results demonstrated that the key cyclization sequence is viable, but we needed to adjust the electronic character of the internal alkene dipolarophile to enhance its reactivity. ${ }^{13}$ Therefore, we chose to add an ester as a mild electron-withdrawing group on the dipolarophile (Scheme 5). ${ }^{2}$ We opted to install the ester group on the internal alkene carbon so that it could advantageously be used to guide the regioselective indoline formation later on. ${ }^{5}$ The synthesis of the new targeted key step substrate began with reduction of nitro 13 followed by alkylation with (iodomethyl)trimethylsilane and formylation ${ }^{11}$ to afford formamide 24. Silyl ether deprotection and Dess-Martin oxidation of the resulting alcohol $\mathbf{2 6}$ gave aldehyde $\mathbf{2 8}$ in excellent yield. The ester was installed using a Baylis-Hillman reaction ${ }^{14}$ followed by benzylation to give the desired substrate 32. ${ }^{15}$ 
Scheme 5. Synthesis of Key Step Substrates 32 and 33

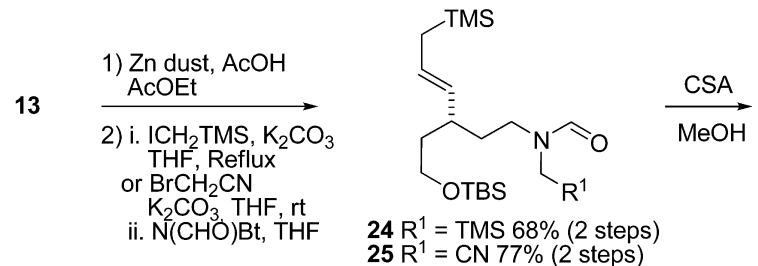

$\overbrace{\mathrm{OH}}^{\mathrm{TMS}}$<smiles>[R1]CN(C=O)CC[C@H](/C=C\CS(C)(=O)=O)CC=O</smiles>

$\mathrm{CH}_{2}=\mathrm{CHCO}_{2} \mathrm{Me}$

$\underset{\text { dioxane/water }}{\stackrel{\mathrm{DABCO}}{\longrightarrow}}$

$26 \mathrm{R}^{1}=\mathrm{TMS} 85 \%$ $27 \mathrm{R}^{1}=\mathrm{CN} 84 \%$

$28 \mathrm{R}^{1}=$ TMS $94 \%$<smiles>[R1]CN(C=O)CC[C@@H](/C=C/C[Y5](C)(C)C)CC(O)C(=C)C(=O)OC</smiles>

$30 \mathrm{R}^{1}=$ TMS $72 \%$ $31 R^{1}=C N 91 \%$
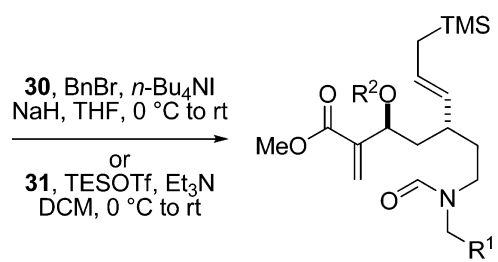

$R^{1}=T M S, R^{2}=B n$ $33 R^{1}=C N, R^{2}=T E S 93 \%$
Formamide 32 was treated with triflic anhydride, and the Vilsmeier-Haack cyclization generated the nonconjugated iminium ion 34 which converted over time (or after flash chromatography) to conjugated iminium ion 35 (Scheme 6).

Scheme 6. Attempted Vilsmeier-Haack/Azomethine Ylide Cycloaddition Sequence with Unsaturated Ester 32

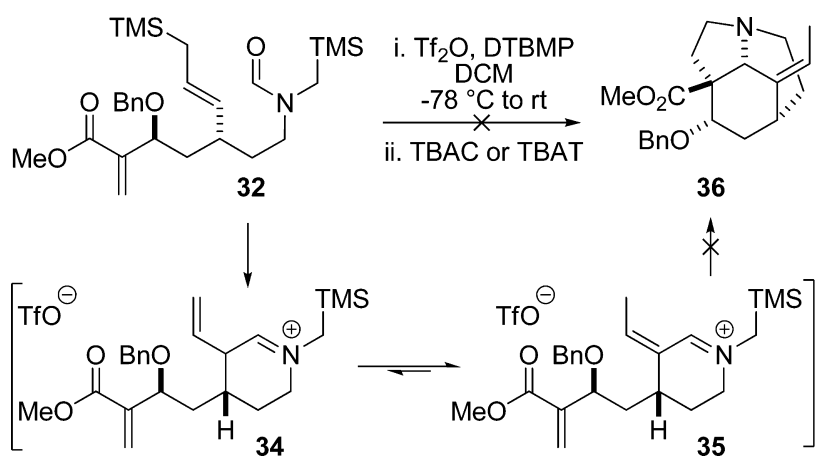

Unfortunately, upon addition of a desilylation agent (TBAC or TBAT) to iminium ion 35, the latter decomposed. We suspected a conjugate addition of the desilylation agent to the unsaturated ester, followed by possible polymerization, as observed with $\mathrm{N}$ phenylmaleimide in the formation of cycloadduct 23 (Scheme 4).

To avoid such side reactions, the obvious solution was to rather prepare the azomethine ylide by deprotonation instead of desilylation. We thus switched to a stabilized azomethine ylide bearing a nitrile. The key step precursor was prepared using the same route described previously (Scheme 5), except that the amine resulting from zinc reduction of nitro 13 was alkylated with bromoacetronitrile. TBS deprotection, oxidation, and Baylis-Hillman reaction ${ }^{14,15}$ generated compound $\mathbf{3 1}$ in yields slightly better than that for the TMS analogue 30. After a TES protection of the free alcohol 31, key step precursor 33 was obtained in only 11 steps. ${ }^{16}$

Subjection of formamide 33 to the Vilsmeier-Haack conditions afforded conjugated iminium ion 39 as confirmed by ${ }^{1} \mathrm{H}$ NMR spectral analysis (Scheme 7$) .{ }^{17}$ In this particular

Scheme 7. Vilsmeier-Haack/Cycloaddition Sequence with Stabilized Azomethine Ylide

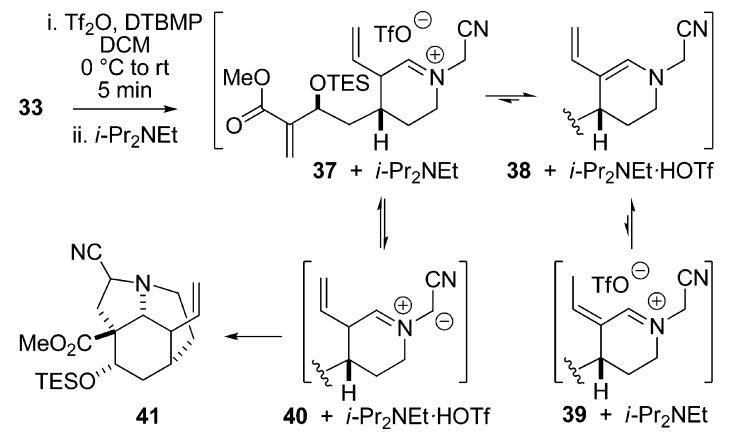

case, tautomerization was fast and we could not observe the nonconjugated iminium ion 37 intermediate. Addition of $i$ $\mathrm{Pr}_{2} \mathrm{NEt}$ at room temperature generated only the cycloadduct 41 bearing a terminal alkene, but in a trace amount. ${ }^{18}$ At first glance, the terminal alkene in $\mathbf{4 1}$ seems inconsistent with the cycloaddition of conjugated iminium ion 39. To explain this result, we postulate that, upon addition of Hunig's base, iminium ion 39 is deprotonated to dienamine 38 , the resting state in solution. Small amounts of protonated iminium ion 37 , kinetically favored over 39 , lead to azomethine ylide 40 , further trapped in the cycloaddition to produce 41 . We could presume that dienamine $\mathbf{3 8}$ is decomposed over time, to account for the poor cycloaddition yield.

To prevent any enolization issue, the allylsilane was replaced with a methyl enol ether (Scheme 8). Hence, Wittig olefination of aldehyde $\mathbf{1 0}$ afforded a separable 3:1 mixture of $E / Z$ isomers. Only the $E$-isomer $\mathbf{4 2}$ was used for the rest of the synthesis. ${ }^{19}$ Following the sequence previously developed, reduction of the nitro group, alkylation with bromoacetonitrile, and in situ formylation ${ }^{11}$ afforded formamide 43 in $76 \%$ overall yield. After

Scheme 8. Synthesis of Key Step Substrate 46
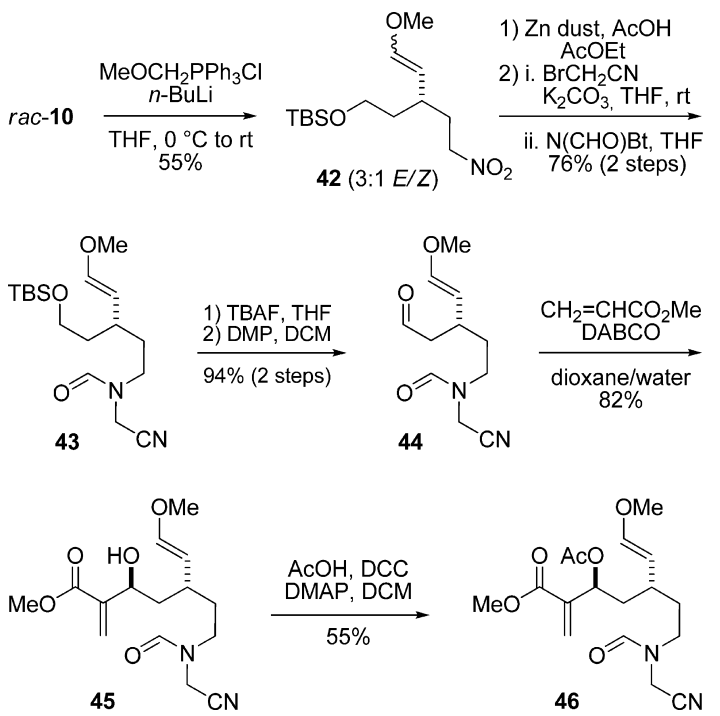
desilylation and oxidation, the resulting aldehyde $\mathbf{4 4}$ was treated under Baylis-Hillman conditions ${ }^{14,15}$ with methyl acrylate to set the dipolarophile. Finally, acetylation ${ }^{20}$ of alcohol 45 provided the key step substrate $\mathbf{4 6}$ in only eight steps from aldehyde $\mathbf{9}$.

Upon activation of formamide 46, the intramolecular Vilsmeier-Haack reaction proceeded in less than $5 \mathrm{~min}$ at room temperature leading to iminium ion 49 (Scheme 9).

Scheme 9. Successful Vilsmeier-Haack/Stabilized Azomethine Ylide Cycloaddition Sequence. ${ }^{23}$
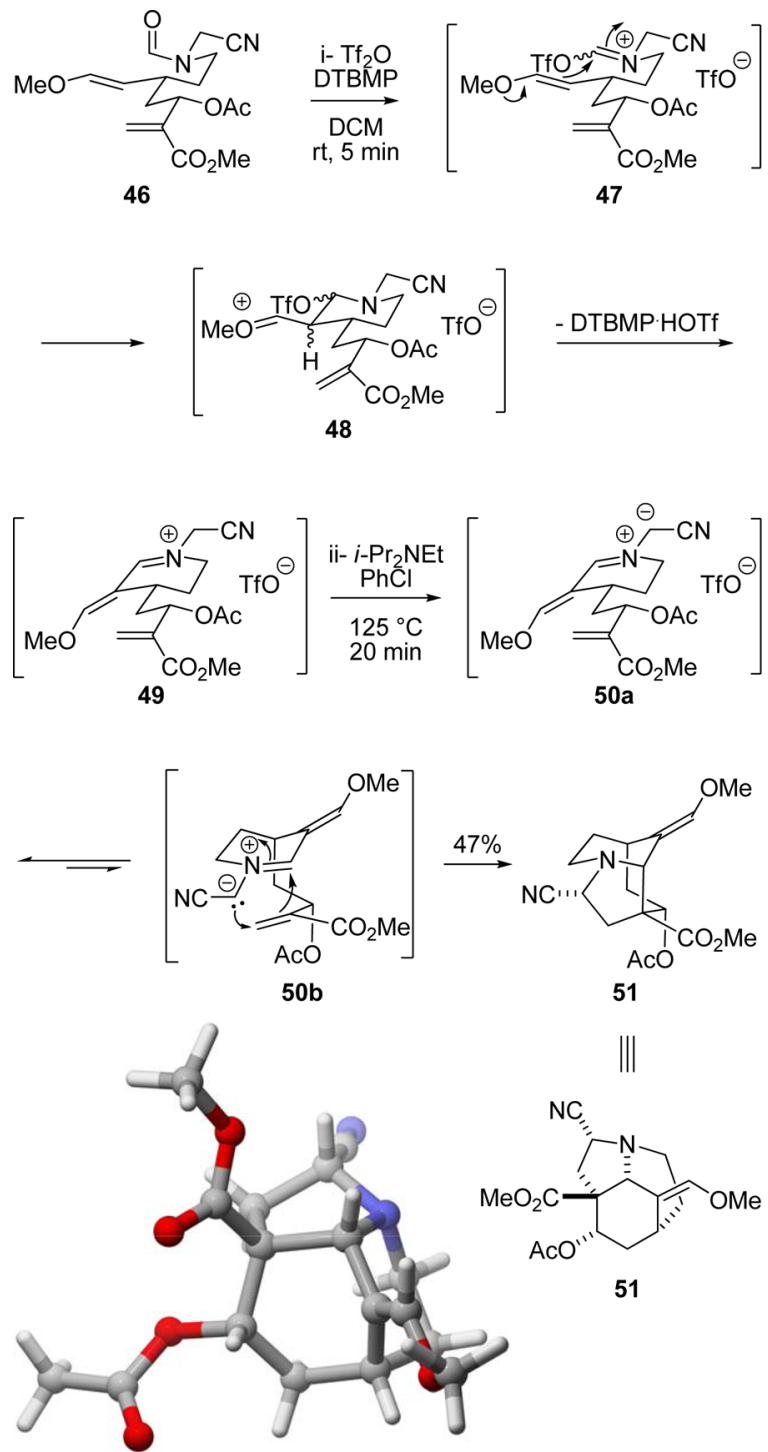

||

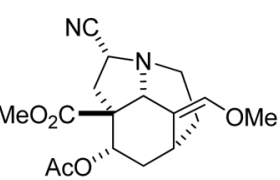

51

Thereafter, the mixture was diluted in chlorobenzene and heated to $125^{\circ} \mathrm{C}$ prior to the addition of $i-\mathrm{PrNEt}_{2}{ }^{21}$ This way, as soon as the deprotonation $\alpha$ to cyano on iminium ion intermediate 49 occurs, the corresponding stabilized azomethine ylide $\mathbf{5 0}$ can adopt the reactive conformation $\mathbf{5 0 b}$ and immediately undergoes intramolecular cycloaddition. The cycloadduct was generated in a mixture of diastereoisomers in only $20 \mathrm{~min}$ and in $47 \%$ yield. Fortunately, one of the diastereomers (51) crystallized out of the mixture and X-ray diffraction confirmed the structure. ${ }^{22}$

The structure of the other diastereomers will need to be confirmed, but data point toward the cis methyl enol ether as the second major diastereomer. Minor diastereomers are usually epimers at the pyrrolidine carbon bearing the nitrile in such reaction cascades. ${ }^{2}$ Fortunately, all these diastereomers will eventually lead to the same common precursor 4 .

\section{CONCLUSION}

In conclusion, we successfully developed a sequential VilsmeierHaack cyclization and intramolecular azomethine ylide cycloaddition strategy applied to synthesis of the core of aspidospermatan alkaloids. This key step proved to be particularly efficient in generating molecular complexity in very short order from a linear precursor prepared in only eight steps. We determined that a methyl enol ether as the nucleophile for the Vilsmeier-Haack cyclization, an unsaturated ester as the dipolarophile, and a stabilized azomethine ylide for the final cycloaddition composed the proper functional group arrangement to ensure perfect chemoselectivity in the one-pot process. Future work will be aimed at finalizing the total synthesis of a series of aspidospermatan alkaloids via functional group manipulations on cycloadduct $\mathbf{5 1}$.,5 $^{4,5}$

\section{EXPERIMENTAL SECTION}

General Information. All reactions requiring anhydrous conditions were conducted in flame-dried glassware under a dry nitrogen or argon atmosphere. Compounds lacking experimental details were prepared according to the literature as cited and are in agreement with published spectra. THF was distilled from $\mathrm{Na}$ and benzophenone at atmospheric pressure. DCM, toluene, diisopropylethylamine, triethylamine, and pyrrolidine were distilled from $\mathrm{CaH}_{2}$ at atmospheric pressure. Triflic anhydride $\left(\mathrm{Tf}_{2} \mathrm{O}\right)$, TFAA, and chlorobenzene were distilled over a small amount of $\mathrm{P}_{2} \mathrm{O}_{5}$ at atmospheric pressure prior to use. Methanol was distilled over $4 \AA$ Å molecular sieves at atmospheric pressure. Zinc powder was activated with two washes of $3 \mathrm{~N} \mathrm{HCl}$, two washes of EtOH, and two washes $\mathrm{Et}_{2} \mathrm{O}$ and then dried under reduced pressure prior to use. 2,6-Ditert-butyl-4-methylpyridine (DTBMP) was synthesized following the reported procedure in literature. ${ }^{24}$ All other required fine chemicals were used directly without purification. Thin layer chromatography (TLC) was conducted with precoated $60 \AA 250 \mu \mathrm{m}$ silica gel plates with F-254 indicator and visualized using a combination of $U V$ and anisaldehyde, ceric ammonium molybdate, iodine on silica, or potassium permanganate staining. Flash column chromatography was performed using silica gel (230-400 mesh). IR spectra were recorded with an FTIR instrument by applying substrates as thin films. ${ }^{1} \mathrm{H}$ and protondecoupled ${ }^{13} \mathrm{C}$ NMR spectra were recorded on $300 \mathrm{MHz}$ and/or 400 $\mathrm{MHz}$ spectrometers. All chemical shifts are referenced to residual nondeutarated solvent. ${ }^{1} \mathrm{H}$ NMR coupling constants are reported in hertz and refer to apparent multiplicities and not true coupling constants. Data for proton spectra are reported as follows: chemical shift (multiplicity [singlet $(\mathrm{s})$, doublet $(\mathrm{d})$, triplet $(\mathrm{t})$, quartet $(\mathrm{q})$, quintet (quint), and multiplet $(\mathrm{m})]$, integration, coupling constants $[\mathrm{Hz}]$ ). Carbon spectra were recorded with complete proton decoupling, and the chemical shifts are reported in ppm. Mass spectra were recorded with an ESI-Q-TOF instrument.

Usual Reaction Workup and Purification. After addition of the indicated aqueous solution, layers were separated. The aqueous phase was extracted with the indicated solvent, and the combined organic phases were washed with the indicated aqueous solution (if needed), dried over anhydrous $\mathrm{MgSO}_{4}$, filtered, and concentrated under reduced pressure using a rotary evaporator. The crude material was purified by flash chromatography using silica gel with the indicated eluent.

(E)-N-(5-Oxo-3-(3-(trimethylsilyl)prop-1-en-1-yl)hept-6-en-1yl)- $N$-((trimethylsilyl)methyl)formamide (6). Dess-Martin periodinane $(172 \mathrm{mg}, 0.41 \mathrm{mmol})$ was added to a solution of $16(120 \mathrm{mg}, 0.34$ $\mathrm{mmol})$ in DCM $(7.0 \mathrm{~mL})$. The reaction mixture was stirred for $4 \mathrm{~h}$, then saturated aqueous $\mathrm{NaHCO}_{3}$ and saturated $\mathrm{Na}_{2} \mathrm{~S}_{2} \mathrm{O}_{3}$ were added, and the mixture was stirred $30 \mathrm{~min}$. The usual workup (DCM) and purification (30\% EtOAc in hexanes) afforded a mixture of rotamers $6(108 \mathrm{mg}$, $91 \%)$ as a colorless oil. ${ }^{1} \mathrm{H}$ NMR $\left(300 \mathrm{MHz}, \mathrm{CDCl}_{3}\right) \delta(\mathrm{ppm}) 7.98(\mathrm{~s})$ and $7.97(\mathrm{~s})(1 \mathrm{H}$, rotamers $), 6.36(\mathrm{~d}, J=10.3 \mathrm{~Hz})$ and $6.30(\mathrm{~d}, J=10.3$ 
$\mathrm{Hz})(1 \mathrm{H}$, rotamers $), 6.18(\mathrm{dd}, 1 \mathrm{H}, J=17.6,1.0 \mathrm{~Hz}), 5.81(\mathrm{dd}, J=10.3$, $1.0 \mathrm{~Hz})$ and $5.80(\mathrm{dd}, J=10.3,1.0 \mathrm{~Hz})(1 \mathrm{H}$, rotamers $), 5.44(\mathrm{td}, 1 \mathrm{H}, J=$ $15.0,7.0 \mathrm{~Hz}), 5.04(\mathrm{dd}, J=15.0,8.0 \mathrm{~Hz})$ and $4.99(\mathrm{dd}, J=15.0,8.0 \mathrm{~Hz})$ $\left(1 \mathrm{H}\right.$, rotamers), $3.19(\mathrm{t}, 2 \mathrm{H}, J=7.0 \mathrm{~Hz}), 2.94$ and $2.68\left(\mathrm{ABq}, J_{A B}=15.0\right.$ $\mathrm{Hz})$ and $2.71(\mathrm{~s})(2 \mathrm{H}$, rotamers $), 2.63-2.51(\mathrm{~m}, 3 \mathrm{H}), 1.77-1.61(\mathrm{~m}$, $1 \mathrm{H}), 1.42-1.40(3 \mathrm{H}, \mathrm{m}), 0.10(\mathrm{~s})$ and $0.08(\mathrm{~s})(9 \mathrm{H}$, rotamers $),-0.03$ (s) and $-0.04(\mathrm{~s})\left(9 \mathrm{H}\right.$, rotamers). ${ }^{13} \mathrm{C} \mathrm{NMR}\left(75 \mathrm{MHz}, \mathrm{CDCl}_{3}\right) \delta(\mathrm{ppm})$ 199.7, 199.2, 162.3, 161.7, 136.9, 136.8, 130.3, 129.9, 129.2, 128.4, 128.3, 128.2, 47.7, 46.1, 45.8, 43.4, 38.6, 37.0, 36.4, 33.9, 33.3, 32.1, 22.9, 22.8, $-1.2,-1.6,-1.8,-1.9$. IR (neat) $\nu\left(\mathrm{cm}^{-1}\right) 2954,2892,1668,1402$, 1247,850 . HRMS (ESI) Calcd for $\mathrm{C}_{18} \mathrm{H}_{35} \mathrm{NO}_{2} \mathrm{Si}_{2} \mathrm{Na}\left[\mathrm{MNa}^{+}\right]$376.2099, found 376.2108 .

4-((tert-Butyldimethylsilyl)oxy)-2-(2-nitroethyl)butanal (10). Following the reported procedure, ${ }^{7}$ a solution of pyrrolidine $(1.8 \mu \mathrm{L}$, $21 \mu \mathrm{mol}), 9^{6}$ (428 mg, $2.11 \mathrm{mmol}$ ), and 2-nitrobenzoic acid (35 mg, 0.21 mmol) in toluene $(1 \mathrm{~mL})$ was cooled to $0{ }^{\circ} \mathrm{C}$ and then nitroethylene (2.4 M in toluene, $441 \mu \mathrm{L}, 1.05 \mathrm{mmol}$ ) was added. The reaction was stirred for 2 days at $3{ }^{\circ} \mathrm{C}$. Saturated aqueous $\mathrm{NH}_{4} \mathrm{Cl}$ was added. The usual workup (EtOAc) and purification (5\% EtOAc in hexanes) afforded $10(202 \mathrm{mg}, 69 \%)$ as a pale yellow oil. ${ }^{1} \mathrm{H}$ NMR $(300 \mathrm{MHz}$, $\left.\mathrm{CDCl}_{3}\right) \delta(\mathrm{ppm}) 9.66(\mathrm{~s}, 1 \mathrm{H}), 4.56-4.38(\mathrm{~m}, 2 \mathrm{H}), 3.70(\mathrm{t}, 2 \mathrm{H}, J=5.7$ $\mathrm{Hz}), 2.59-2.52(\mathrm{~m}, 1 \mathrm{H}), 2.43-2.31(\mathrm{~m}, 1 \mathrm{H}), 2.15-2.08(\mathrm{~m}, 1 \mathrm{H})$, $2.01-1.90(\mathrm{~m}, 1 \mathrm{H}), 1.89-1.79(\mathrm{~m}, 1 \mathrm{H}) 0.86(\mathrm{~s}, 9 \mathrm{H}), 0.03(\mathrm{~s}, 6 \mathrm{H}) .{ }^{13} \mathrm{C}$ $\operatorname{NMR}\left(75 \mathrm{MHz}, \mathrm{CDCl}_{3}\right) \delta(\mathrm{ppm}) 202.6(\mathrm{~d}), 73.5(\mathrm{t}), 60.0(\mathrm{t}), 46.2(\mathrm{~d})$, $32.0(\mathrm{t}), 26.0(\mathrm{q}), 25.6(\mathrm{t}), 18.3(\mathrm{~s}),-5.4(\mathrm{q})$. IR (neat) $\nu\left(\mathrm{cm}^{-1}\right) 2929$, 2857, 1723, 1551, 1252, 834. HRMS (ESI) Calcd for $\mathrm{C}_{12} \mathrm{H}_{25} \mathrm{NO}_{4} \mathrm{SiNa}$ $\left[\mathrm{MNa}^{+}\right]$298.1445, found 298.1449.

6-((tert-Butyldimethylsilyl)oxy)-4-(2-nitroethyl)hex-1-en-3-ol (11). Vinylmagnesium bromide (1.0 M in THF, $6.8 \mathrm{~mL}, 6.80 \mathrm{mmol}$ ) was added dropwise to a solution of $10(1.30 \mathrm{~g}, 4.72 \mathrm{mmol})$ in THF $(50 \mathrm{~mL})$ at $-78{ }^{\circ} \mathrm{C}$. The mixture was stirred for $20 \mathrm{~min}$ at $-78^{\circ} \mathrm{C}$ and then 20 min at rt. Saturated aqueous $\mathrm{NH}_{4} \mathrm{Cl}$ was added. The usual workup $\left(\mathrm{Et}_{2} \mathrm{O}\right)$ and purification (10 to $15 \%$ EtOAc in hexanes) afforded an inseparable mixture of diastereomers $11(912 \mathrm{mg}, 64 \%)$ as a colorless oil. ${ }^{1} \mathrm{H}$ NMR $\left(300 \mathrm{MHz}, \mathrm{CDCl}_{3}\right) \delta(\mathrm{ppm}) 5.83(\mathrm{ddd}, 1 \mathrm{H}, J=17.0,10.5,5.6$ $\mathrm{Hz}), 5.27(\mathrm{ddt}, 1 \mathrm{H}, J=17.0,5.6,1.5 \mathrm{~Hz}), 5.21(\mathrm{dq}, 1 \mathrm{H}, J=10.5,1.5 \mathrm{~Hz})$, $4.50-4.41(\mathrm{~m}, 2 \mathrm{H}), 4.12(\mathrm{dt}, 1 \mathrm{H}, J=10.5,4.7 \mathrm{~Hz}), 3.75-3.62(\mathrm{~m}, 2 \mathrm{H})$, 3.03 (br. s, $1 \mathrm{H}), 2.26-2.10(\mathrm{~m}, 1 \mathrm{H}), 2.04-1.91(\mathrm{~m}, 1 \mathrm{H}), 1.83-1.67(\mathrm{~m})$ and $1.64-1.47(\mathrm{~m})(3 \mathrm{H}), 0.88(\mathrm{~s}, 9 \mathrm{H}), 0.05(\mathrm{~s}, 6 \mathrm{H}) .{ }^{13} \mathrm{C}$ NMR $(75$ $\left.\mathrm{MHz}, \mathrm{CDCl}_{3}\right) \delta(\mathrm{ppm}) 138.9(\mathrm{~d}), 138.9(\mathrm{~d}), 116.3(\mathrm{t}), 116.1(\mathrm{t}), 74.5$ $(\mathrm{t}), 74.4(\mathrm{t}), 74.4(\mathrm{t}), 74.3(\mathrm{t}), 61.2(\mathrm{t}), 60.6(\mathrm{t}), 39.0(\mathrm{~d}), 38.7(\mathrm{~d}), 33.4$ $(\mathrm{t}), 32.3(\mathrm{t}), 28.3(\mathrm{t}), 27.7(\mathrm{t}), 26.0(\mathrm{q}), 26.0(\mathrm{q}), 18.4(\mathrm{~s}), 18.3(\mathrm{~s}),-5.4$ (q), -5.4 (q). IR (neat) $\nu\left(\mathrm{cm}^{-1}\right)$ 2953, 2929, 2857, 1725, 1550, 1384, 1254, 1092, 834. HRMS (ESI) Calcd for $\mathrm{C}_{14} \mathrm{H}_{29} \mathrm{NO}_{4} \mathrm{SiNa}\left[\mathrm{MNa}^{+}\right]$ 326.1758 , found 326.1761 .

6-((tert-Butyldimethylsilyl)oxy)-4-(2-nitroethyl)hex-1-en-3-yl 2,2,2-trifluoroacetate (12). TFAA $(1.00 \mathrm{~mL}, 7.07 \mathrm{mmol})$ was added to a solution of $11(1.43 \mathrm{~g}, 4.71 \mathrm{mmol})$, pyridine $(0.57 \mathrm{~mL}, 7.07 \mathrm{mmol})$, and DMAP (115 mg, $0.94 \mathrm{mmol})$ in DCM $(50 \mathrm{~mL})$ at $0{ }^{\circ} \mathrm{C}$. The reaction mixture was stirred for $20 \mathrm{~min}$ at $0^{\circ} \mathrm{C}$. After $1 \mathrm{~h}$ at $\mathrm{rt}$, water was added. The usual workup (DCM) and purification (5\% EtOAc in hexanes) afforded 12 (1.57 g, 84\%) as a yellow oil. ${ }^{1} \mathrm{H}$ NMR $(300 \mathrm{MHz}$, $\left.\mathrm{CDCl}_{3}\right) \delta(\mathrm{ppm}) 5.81(\mathrm{ddd}, 1 \mathrm{H}, J=17.0,10.7,6.0 \mathrm{~Hz}), 5.56-5.49(\mathrm{~m}$, $1 \mathrm{H}), 5,41(\mathrm{t}, 1 \mathrm{H}, J=6.0 \mathrm{~Hz}), 5.38-5.32(\mathrm{~m}, 1 \mathrm{H}), 4.51-4.44(\mathrm{~m}, 2 \mathrm{H})$, $3,71-3.66(\mathrm{~m}, 2 \mathrm{H}), 2.22-2.12(\mathrm{~m}, 1 \mathrm{H}), 2.09-1.99(\mathrm{~m}, 2 \mathrm{H}), 1.73-1.61$ $(\mathrm{m}, 1 \mathrm{H}), 1.59-1.47(\mathrm{~m}, 1 \mathrm{H}), 0.88(\mathrm{~s}, 9 \mathrm{H}), 0.05(\mathrm{~s}, 6 \mathrm{H}) .{ }^{19} \mathrm{~F}$ NMR $(282$ $\left.\mathrm{MHz}, \mathrm{CDCl}_{3}\right) \delta(\mathrm{ppm})-75.13,-75.19 .{ }^{13} \mathrm{C} \mathrm{NMR}\left(75 \mathrm{MHz} \mathrm{CDCl}_{3}\right) \delta$ $(\mathrm{ppm}) 156.6\left(\mathrm{qd}, J_{\mathrm{C}-\mathrm{F}}=42.2 \mathrm{~Hz}, \mathrm{~s}\right), 132.0(\mathrm{~d}), 131.0(\mathrm{~d}), 120.8(\mathrm{t})$, $120.2(\mathrm{t}), 114.7\left(\mathrm{q}, J_{\mathrm{C}-\mathrm{F}}=284.0 \mathrm{~Hz}\right), 80.8(\mathrm{~d}), 80.6(\mathrm{~d}), 73.6(\mathrm{t}), 60.6$ $(\mathrm{t}), 60.2(\mathrm{t}), 36.6(\mathrm{~d}), 36.2(\mathrm{~d}), 32.8(\mathrm{t}), 32.6(\mathrm{t}), 27.8(\mathrm{t}), 27.2(\mathrm{t}), 26.0$ (q), 18.3 (q), -5.4 (q). IR (neat) $\nu\left(\mathrm{cm}^{-1}\right) 2954,2930,2858,1784$, $1555,1220,1153,834$. HRMS (ESI) Calcd for $\mathrm{C}_{16} \mathrm{H}_{28} \mathrm{~F}_{3} \mathrm{NO}_{5} \mathrm{SiNa}$ $\left[\mathrm{MNa}^{+}\right]$422.1581, found 422.1583 .

(E)-tert-Butyldimethyl((3-(2-nitroethyl)-6-(trimethylsilyl)hex4-en-1-yl)oxy)silane (13). Following the reported procedure, ${ }^{8}$ a solution of $12(3.02 \mathrm{~g}, 7.56 \mathrm{mmol})$ in THF $(10 \mathrm{~mL})$ was added to a solution of hexamethyldisilane $(3.10 \mathrm{~mL}, 15.1 \mathrm{mmol})$ and bis(dibenzylideneacetone)palladium $(131 \mathrm{mg}, 0.23 \mathrm{mmol})$ in THF $(40$ $\mathrm{mL}$ ) at $\mathrm{rt}$. The solution was stirred for $15 \mathrm{~h}$ at $\mathrm{rt}$, and then saturated aqueous $\mathrm{NaHCO}_{3}$ was added. The usual workup $\left(\mathrm{Et}_{2} \mathrm{O}\right)$ and purification ( $3 \%$ EtOAc in hexanes) gave $13(2.58 \mathrm{~g}, 95 \%)$ as a colorless oil. ${ }^{1} \mathrm{H}$ NMR $\left(300 \mathrm{MHz}, \mathrm{CDCl}_{3}\right) \delta(\mathrm{ppm}) 5.43(\mathrm{dt}, 1 \mathrm{H}, J=$ $15.5,8.0 \mathrm{~Hz}), 4.91(\mathrm{dd}, 1 \mathrm{H}, J=15.5,9.0 \mathrm{~Hz}), 4.36(\mathrm{dd}, 2 \mathrm{H}, J=7.5,6.8$ $\mathrm{Hz}$ ), 3.67-3.51 (m, $2 \mathrm{H}), 2.21-2.09(\mathrm{~m}, 2 \mathrm{H}), 1.86-1.73(\mathrm{~m}, 1 \mathrm{H})$, $1.67-1.55(\mathrm{~m}, 1 \mathrm{H}), 1.54-1.47(\mathrm{~m}, 1 \mathrm{H}), 1.44(\mathrm{dd}, 2 \mathrm{H}, J=8.1,1.1 \mathrm{~Hz})$, $0.88(\mathrm{~s}, 9 \mathrm{H}), 0.03(\mathrm{~s}, 6 \mathrm{H}), 0.00(\mathrm{~s}, 9 \mathrm{H}) .{ }^{13} \mathrm{C} \mathrm{NMR}\left(75 \mathrm{MHz}, \mathrm{CDCl}_{3}\right) \delta$ $(\mathrm{ppm}) 130.0(\mathrm{~d}), 129.6(\mathrm{~d}), 74.3(\mathrm{t}), 60.9(\mathrm{t}), 38.7(\mathrm{t}), 37.4(\mathrm{~d}), 33.1(\mathrm{t})$, $26.1(\mathrm{q}), 23.0(\mathrm{t}), 18.4(\mathrm{~s}), 1.8(\mathrm{q}),-5.2(\mathrm{q})$. IR (neat) $\nu\left(\mathrm{cm}^{-1}\right) 2953$, $2928,2857,1552,1248,1100$. HRMS (ESI) Calcd for $\mathrm{C}_{17} \mathrm{H}_{37} \mathrm{NO}_{3} \mathrm{Si}_{2} \mathrm{Na}$ $\left[\mathrm{MNa}^{+}\right]$382.2204, found 382.2209.

(E)-3-(2-Nitroethyl)-6-(trimethylsilyl)hex-4-enal (14). Camphorsulfonic acid $(2.00 \mathrm{~g}, 8.60 \mathrm{mmol})$ was added to a solution of 13 $(2.58 \mathrm{~g}, 7.17 \mathrm{mmol})$ in $\mathrm{MeOH}(70 \mathrm{~mL})$. The reaction mixture was stirred for $30 \mathrm{~min}$, and then water was added. The usual workup (EtOAc) and purification (20\% EtOAc in hexanes) afforded the corresponding alcohol $(1.65 \mathrm{~g}, 94 \%)$ as a colorless oil. ${ }^{1} \mathrm{H}$ NMR (300 $\left.\mathrm{MHz}, \mathrm{CDCl}_{3}\right) \delta(\mathrm{ppm}) 5.49(\mathrm{dt}, 1 \mathrm{H}, J=15.0,8.0 \mathrm{~Hz}), 4.93(\mathrm{dd}, 1 \mathrm{H}, J=$ 15.0, 9.0), 4.37 (dd, $2 \mathrm{H}, J=7.5,6.5 \mathrm{~Hz}), 3.73-3.59(\mathrm{~m}, 2 \mathrm{H}), 2.25-2.13$ $(\mathrm{m}, 2 \mathrm{H}), 1.87-1.74(\mathrm{~m}, 1 \mathrm{H}), 1.72-1.62(\mathrm{~m}, 1 \mathrm{H}), 1.58-1.51(\mathrm{~m}, 1 \mathrm{H})$, $1.45(\mathrm{~d}, 2 \mathrm{H}, J=8.0,1.0 \mathrm{~Hz}), 1.27$ (br. s, $1 \mathrm{H}), 0.01(\mathrm{~s}, 9 \mathrm{H}) .{ }^{13} \mathrm{C}$ NMR $(75$ $\left.\mathrm{MHz}, \mathrm{CDCl}_{3}\right) \delta(\mathrm{ppm}) 130.3,129.8,74.1,60.9,38.5,37.8,33.1,23.1$, -1.8. IR (neat) $\nu\left(\mathrm{cm}^{-1}\right)$ 2952, 2901, 1549, 1379, 1246, 846. HRMS (ESI) Calcd for $\mathrm{C}_{11} \mathrm{H}_{23} \mathrm{NO}_{3} \mathrm{SiNa}\left[\mathrm{MNa}^{+}\right]$268.1339, found 268.1342. Following the procedure used to prepare 6 , the alcohol ( $(1.65 \mathrm{~g}, 6.72$ $\mathrm{mmol})$ in DCM $(130 \mathrm{~mL})$ was treated with Dess-Martin periodinane $(3.42 \mathrm{~g}, 8.07 \mathrm{mmol})$ for $2 \mathrm{~h}$. The usual workup (DCM) and purification (10\% EtOAc in hexanes) afforded $14(1.54 \mathrm{~g}, 94 \%)$ as a colorless oil. ${ }^{1} \mathrm{H}$ $\operatorname{NMR}\left(300 \mathrm{MHz}, \mathrm{CDCl}_{3}\right) \delta(\mathrm{ppm}) 9.69(\mathrm{t}, 1 \mathrm{H}, J=2.0 \mathrm{~Hz}), 5.55(\mathrm{dt}, 1 \mathrm{H}$, $J=15.5,8.0 \mathrm{~Hz}), 4.99(\mathrm{dd}, 1 \mathrm{H}, J=15.5,9.0 \mathrm{~Hz}), 4.39(\mathrm{dd}, 2 \mathrm{H}, J=7.5,6.5$ $\mathrm{Hz}), 2.72-2.58(\mathrm{~m}, 1 \mathrm{H}), 2.46(\mathrm{dd}, 2 \mathrm{H}, J=6.9,2.0 \mathrm{~Hz}), 2.26-2.15(\mathrm{~m}$, $1 \mathrm{H}), 1.92-1.80(\mathrm{~m}, 1 \mathrm{H}), 1.44(\mathrm{dd}, 2 \mathrm{H}, J=8.0,1.0 \mathrm{~Hz}), 0.01(\mathrm{~s}, 9 \mathrm{H}) .{ }^{13} \mathrm{C}$ NMR $\left(75 \mathrm{MHz}, \mathrm{CDCl}_{3}\right) \delta(\mathrm{ppm})$ 201.1, 131.2, 128.0, 73.6, 49.3, 35.4, 32.3, 23.1, -1.8. IR (neat) $\nu\left(\mathrm{cm}^{-1}\right) 2953,2896,1723,1549,1379,1246$, 848. HRMS (ESI) Calcd for $\mathrm{C}_{12} \mathrm{H}_{27} \mathrm{NO}_{4} \mathrm{SiNa}\left[\mathrm{M}+\mathrm{MeOH}+\mathrm{Na}^{+}\right]$ 298.1445 , found 298.1457.

(E)-5-(2-Nitroethyl)-8-(trimethylsilyl)octa-1,6-dien-3-ol (15). Following the procedure used to prepare $11,14(1.54 \mathrm{~g}, 6.33 \mathrm{mmol})$ in THF $(65 \mathrm{~mL})$ was treated with vinylmagnesium bromide $(1.0 \mathrm{M}$ in THF, $9.50 \mathrm{~mL}, 9.49 \mathrm{mmol}$ ) for $40 \mathrm{~min}$ at $-78^{\circ} \mathrm{C}$ and then for $1 \mathrm{~h}$ at rt. The usual workup $\left(\mathrm{Et}_{2} \mathrm{O}\right)$ and purification (12.5 to $20 \%$ EtOAc in hexanes) afforded a separable 1:1 mixture of diastereomers 15 (1.49 $\mathrm{g}$, $87 \%)$ as a colorless oil. Diastereomer 1: ${ }^{1} \mathrm{H} \mathrm{NMR}\left(300 \mathrm{MHz}, \mathrm{CDCl}_{3}\right) \delta$ (ppm) $5.87(\mathrm{ddd}, 1 \mathrm{H}, J=17.0,10.4,6.0 \mathrm{~Hz}), 5.53(\mathrm{dt}, 1 \mathrm{H}, J=15.0,8.0$ $\mathrm{Hz}), 5.21(\mathrm{dt}, 1 \mathrm{H}, J=17.0,1.5 \mathrm{~Hz}), 5.09(\mathrm{dt}, 1 \mathrm{H}, J=10.4,1.5 \mathrm{~Hz}), 4.91$ $(\mathrm{ddt}, 1 \mathrm{H}, J=15.0,9.2,1.1 \mathrm{~Hz}), 4.37(\mathrm{dd}, 2 \mathrm{H}, J=8.0,6.6 \mathrm{~Hz}), 4.19-4.11$ $(\mathrm{m}, 1 \mathrm{H}), 2.39-2.26(\mathrm{~m}, 1 \mathrm{H}), 2.21-2.09(\mathrm{~m}, 1 \mathrm{H}), 1.87-1.74(\mathrm{~m}, 1 \mathrm{H})$, $1.58-1.43(\mathrm{~m}, 5 \mathrm{H}), 0.01(\mathrm{~s}, 9 \mathrm{H}) .{ }^{13} \mathrm{C} \mathrm{NMR}\left(75 \mathrm{MHz} \mathrm{CDCl}_{3}\right) \delta(\mathrm{ppm})$ $141.4,130.6,129.6,114.4,74.1,70.6,43.0,37.3,33.3,23.1,-1.8$. IR (neat) $\nu\left(\mathrm{cm}^{-1}\right) 3381,2951,2919,1549,1430,1375,1247,991,844$. HRMS (ESI) Calcd for $\mathrm{C}_{13} \mathrm{H}_{25} \mathrm{NO}_{3} \mathrm{SiNa}\left[\mathrm{MNa}^{+}\right] 294.1495$, found 294.1499. Diastereomer 2: ${ }^{1} \mathrm{H}$ NMR $\left(300 \mathrm{MHz}, \mathrm{CDCl}_{3}\right) \delta$ (ppm) 5.81 (ddd, $1 \mathrm{H}, J=17.0,10.3,6.5 \mathrm{~Hz}), 5.46(\mathrm{dt}, 1 \mathrm{H}, J=15.0,8.0 \mathrm{~Hz}), 5.21(\mathrm{dt}$, $1 \mathrm{H}, J=17.0,1.0 \mathrm{~Hz}), 5.13(\mathrm{dt}, 1 \mathrm{H}, J=10.3,1.0 \mathrm{~Hz}), 4.98(\mathrm{ddt}, 1 \mathrm{H}, J=$ $15.0,9.0,1.0 \mathrm{~Hz}), 4.36(\mathrm{dd}, 2 \mathrm{H}, J=8.0,6.4 \mathrm{~Hz}), 4.20-4.13(\mathrm{~m}, 1 \mathrm{H})$, $2.26-2.07(\mathrm{~m}, 2 \mathrm{H}), 1.85-1.75(\mathrm{~m}, 1 \mathrm{H}), 1.68-1.50(\mathrm{~m}, 3 \mathrm{H}), 1.46(\mathrm{dd}$, $2 \mathrm{H}, J=8.0,1.0 \mathrm{~Hz}), 0.01(\mathrm{~s}, 9 \mathrm{H}) .{ }^{13} \mathrm{C} \mathrm{NMR}\left(75 \mathrm{MHz} \mathrm{CDCl}_{3}\right) \delta(\mathrm{ppm})$ $140.6,130.2,130.1,115.7,73.9,71.7,42.9,37.8,32.9,23.1,-1.7$. IR (neat) $\nu\left(\mathrm{cm}^{-1}\right) 3386,2955,2919,1545,1426,1375,1247,991,844$. HRMS (ESI) Calcd for $\mathrm{C}_{13} \mathrm{H}_{25} \mathrm{NO}_{3} \mathrm{SiNa}\left[\mathrm{MNa}^{+}\right]$294.1496, found 294.1487.

(E)-N-(5-Hydroxy-3-(3-(trimethylsilyl)prop-1-en-1-yl)hept-6en-1-yl)- $N$-((trimethylsilyl)methyl)formamide (16). Following the reported procedure for reduction of the nitro group, ${ }^{10}$ acetic acid (1.00 $\mathrm{mL}, 18.4 \mathrm{mmol})$ and activated zinc powder $(1.20 \mathrm{~g}, 18.4 \mathrm{mmol})$ were added to a solution of 15 (500 mg, $1.84 \mathrm{mmol})$ in EtOAc $(10 \mathrm{~mL})$. After $1 \mathrm{~h}$ at $\mathrm{rt}$, the mixture was filtered through a Celite pad and the latter was washed with EtOAc. The filtrate was concentrated under reduced pressure, and the crude was diluted with hexanes and then concentrated (three cycles) in order to remove the remaining acetic acid. $\mathrm{K}_{2} \mathrm{CO}_{3}(2.55$ $\mathrm{g}, 18.4 \mathrm{mmol})$ and (iodomethyl)trimethylsilane $(0.36 \mathrm{~mL}, 2.39 \mathrm{mmol})$ 
were added to the crude amine in THF $(10 \mathrm{~mL})$. The reaction mixture was refluxed for $20 \mathrm{~h}$ and then was allowed to cool before being filtering. $N$-Formylbenzotriazole $(300 \mathrm{mg}, 1.84 \mathrm{mmol})$ was added to the filtrate, and the reaction mixture was stirred for $15 \mathrm{~h}$ at $\mathrm{rt}$. The mixture was concentrated. The usual purification (12.5 to $20 \%$ EtOAc in DCM) afforded a mixture of rotamers $16(411 \mathrm{mg}, 63 \%)$ as a colorless oil. ${ }^{1} \mathrm{H}$ NMR $\left(300 \mathrm{MHz}, \mathrm{CDCl}_{3}\right) \delta(\mathrm{ppm}) 7.97(\mathrm{~s})$ and $7.96(\mathrm{~s})(1 \mathrm{H}$, rotamers), 5.79 (ddd, $1 \mathrm{H}, J=17.0,10.3,6.7 \mathrm{~Hz}), 5.39(\mathrm{dt}, 1 \mathrm{H}, J=15.5$, $8.0 \mathrm{~Hz}), 5.18(\mathrm{dt}, 1 \mathrm{H}, J=17.0,1.5 \mathrm{~Hz}), 5.10(\mathrm{dt}, 1 \mathrm{H}, J=10.3,1.5 \mathrm{~Hz})$, $5.07(\mathrm{ddt}, J=15.5,9.0,1.0 \mathrm{~Hz})$ and $4.99(\mathrm{ddt}, J=15.5,9.0,1.0 \mathrm{~Hz})(1 \mathrm{H}$, rotamers), $4.17-4.10(\mathrm{~m}, 1 \mathrm{H}), 3.33-3.22(\mathrm{~m})$ and $3.17(\mathrm{t}, J=7.3 \mathrm{~Hz})$ $(2 \mathrm{H}$, rotamers $), 2.90$ and $2.66\left(\mathrm{ABq}, J_{\mathrm{AB}}=15.0 \mathrm{~Hz}\right)$ and $2.67(\mathrm{~s})(2 \mathrm{H}$, rotamers), $2.07-1.95(\mathrm{~m}, 1 \mathrm{H}), 1.79($ br. s, $1 \mathrm{H}), 1.70-1.31(\mathrm{~m}, 6 \mathrm{H})$, $0.09(\mathrm{~s})$ and $0.07(\mathrm{~s})(9 \mathrm{H}$, rotamers $),-0.02(\mathrm{~s}, 9 \mathrm{H}) .{ }^{13} \mathrm{C}$ NMR $(75$ $\mathrm{MHz}, \mathrm{CDCl}_{3}$ ) $\delta(\mathrm{ppm}) 162.3$ (d), 161.8 (d), 141.0 (d), 140.8 (d), 132.1 (d), $131.5(\mathrm{~d}), 128.9(\mathrm{~d}), 128.2(\mathrm{~d}), 115.5(\mathrm{t}), 115.2(\mathrm{t}), 71.9(\mathrm{~d}), 71.8$ (d), $47.7(\mathrm{t}), 43.6(\mathrm{t}), 43.22(\mathrm{t}), 43.17(\mathrm{t}), 38.8,38.3,37.6(\mathrm{~d}), 34.1(\mathrm{t})$, $34.0(\mathrm{t}), 32.6(\mathrm{t}), 23.0(\mathrm{t}), 22.9(\mathrm{t}),-1.2(\mathrm{q}),-1.7(\mathrm{q}),-1.9(\mathrm{q})$. IR (neat) $\nu\left(\mathrm{cm}^{-1}\right) 3376,2952,1655,1389,1246,840$. HRMS (ESI) Calcd for $\mathrm{C}_{18} \mathrm{H}_{37} \mathrm{NO}_{2} \mathrm{Si}_{2} \mathrm{Na}\left[\mathrm{MNa}^{+}\right]$378.2255, found 378.2265.

(E)-N-(5-(Benzyloxy)-3-(3-(trimethylsilyl)prop-1-en-1-yl)hept-6-en-1-yl)- $N$-((trimethylsilyl)methyl)formamide (19). $\mathrm{NaH}$ (60\% dispersion in mineral oil, $92.4 \mathrm{mg}, 2.31 \mathrm{mmol}$ ) was added to a solution of $16(411 \mathrm{mg}, 1.16 \mathrm{mmol})$ in THF $(10 \mathrm{~mL})$ at $0{ }^{\circ} \mathrm{C} . n-\mathrm{Bu}_{4} \mathrm{NI}$ $(427 \mathrm{mg}, 1.16 \mathrm{mmol})$ and benzyl bromide $(1.37 \mathrm{~mL}, 11.6 \mathrm{mmol}$, freshly distilled) were successively added, and the mixture was stirred for $20 \mathrm{~h}$ at rt. Saturated aqueous $\mathrm{NaHCO}_{3}$ was added. The usual workup $\left(\mathrm{Et}_{2} \mathrm{O}\right)$ and purification (20\% EtOAc in hexanes) afforded a mixture of rotamers $19(435 \mathrm{mg}, 84 \%)$ as a colorless oil. ${ }^{1} \mathrm{H}$ NMR $\left(300 \mathrm{MHz}, \mathrm{CDCl}_{3}\right) \delta$ (ppm) $7.89(\mathrm{~s}, 1 \mathrm{H}), 7.32-7.21(\mathrm{~m}, 5 \mathrm{H}), 5.62(\mathrm{ddd}, 1 \mathrm{H}, J=17.5,10.0$, $8.0 \mathrm{~Hz}), 5.30(\mathrm{dt}, 1 \mathrm{H}, J=15.5,8.0 \mathrm{~Hz}), 5.22(\mathrm{dd}, 1 \mathrm{H}, J=10.0,1.5 \mathrm{~Hz})$, $5.15(\mathrm{dd}, 1 \mathrm{H}, J=17.0,1.5 \mathrm{~Hz}), 4.95(\mathrm{~d}, J=9.0 \mathrm{~Hz})$ and $4.87(\mathrm{dd}, J=15.0$, $9.0 \mathrm{~Hz})(1 \mathrm{H}), 4.38$ and $4.37\left(\mathrm{ABq}, 2 \mathrm{H}, J_{A B}=12.0 \mathrm{~Hz}\right), 3.77-3.69(\mathrm{~m}$, $1 \mathrm{H}), 3.23-3.14(\mathrm{~m})$ and $3.08(\mathrm{t}, J=7.0 \mathrm{~Hz})(2 \mathrm{H}), 2.82$ and $2.58(\mathrm{ABq}$, $\left.J_{\mathrm{AB}}=15.0 \mathrm{~Hz}\right)$ and $2.62(\mathrm{~s})(2 \mathrm{H}), 2.03-1.90(\mathrm{~m}, 1 \mathrm{H}), 1.69-1.26(\mathrm{~m}$, $6 \mathrm{H}),-0.03(\mathrm{~s})$ and $-0.02(\mathrm{~s})(9 \mathrm{H}),-0.07(\mathrm{~s}, 9 \mathrm{H}) .{ }^{13} \mathrm{C} \mathrm{NMR}(75 \mathrm{MHz}$, $\left.\mathrm{CDCl}_{3}\right) \delta(\mathrm{ppm}) 162.2,161.8,138.9,138.8,138.7,138.7,131.8,131.4$, $128.7,128.5,128.4,127.9,127.9,127.8,127.7,127.6,127.5,127.1,118.0$, 117.9, 79.1, 78.8, 70.1, 65.4, 65.4, 47.7, 43.6, 41.5, 41.4, 38.6, 37.5, 36.7, 34.1, 33.9, 32.5, 22.9, 22.8, $-1.2,-1.7,-1.8$. IR (neat) $\nu\left(\mathrm{cm}^{-1}\right) 2954$, 2927, 2896, 2862, 1666, 1246. HRMS (ESI) Calcd for $\mathrm{C}_{25} \mathrm{H}_{43} \mathrm{NO}_{2} \mathrm{Si}_{2} \mathrm{Na}$ $\left[\mathrm{MNa}^{+}\right]$468.2725, found 468.2737.

4-(2-(Benzyloxy)but-3-en-1-yl)-1-((trimethylsilyl)methyl)-5vinyl-2,3,4,5-tetrahydropyridin-1-ium trifluoromethanesulfonate (20) and 4-(2-(Benzyloxy)but-3-en-1-yl)-5-ethylidene-1-((trimethylsilyl)methyl)-2,3,4,5-tetrahydropyridin-1ium Trifluoromethanesulfonate (21). $\mathrm{Tf}_{2} \mathrm{O}(32 \mu \mathrm{L}, 0.19 \mathrm{mmol})$ was added to a solution of $19(76.0 \mathrm{mg}, 0.17 \mathrm{mmol})$ and DTBMP $(38.5 \mathrm{mg}$, $0.19 \mathrm{mmol})$ in DCM $(3.5 \mathrm{~mL})$ at $-78^{\circ} \mathrm{C}$. After $15 \mathrm{~min}$ at $-78^{\circ} \mathrm{C}$ and then $45 \mathrm{~min}$ at $\mathrm{rt},{ }^{1} \mathrm{H}$ NMR analysis of an aliquot showed the formation of the iminium ion 20: ${ }^{1} \mathrm{H}$ NMR $\left(300 \mathrm{MHz}, \mathrm{CD}_{2} \mathrm{Cl}_{2}\right)$ characteristic signals $\delta(\mathrm{ppm}) 8.19(\mathrm{~s}), 7.36-7.30(\mathrm{~m}, 5 \mathrm{H}), 5.81-5.64(\mathrm{~m}, 2 \mathrm{H}), 5.52-$ $5.45(\mathrm{~m}, 1 \mathrm{H}), 5.32-5.26(\mathrm{~m}, 3 \mathrm{H}), 4.68(\mathrm{~s})$, and 4.60 and $4.28\left(\mathrm{ABq}, J_{\mathrm{AB}}\right.$ $=12.0 \mathrm{~Hz})(2 \mathrm{H}), 3.88-3.75(\mathrm{~m} 2 \mathrm{H}), 3.65-3.46(\mathrm{~m})$ and $3.31-3.26$ (m) $(3 \mathrm{H}), 0.22(\mathrm{~s}, 9 \mathrm{H})$. The reaction mixture was concentrated. The usual purification (0 to $10 \% \mathrm{MeOH}$ in EtOAc) afforded $21(65.0 \mathrm{mg}$, $76 \%)$ as a pale yellow oil. ${ }^{1} \mathrm{H}$ NMR $\left(300 \mathrm{MHz}, \mathrm{CDCl}_{3}\right)$ characteristic signals $\delta(\mathrm{ppm}) 8.61(\mathrm{~s}, 1 \mathrm{H}), 7.36-7.26(\mathrm{~m}, 5 \mathrm{H}), 7.09(\mathrm{q}, 1 \mathrm{H}, J=7.0$ $\mathrm{Hz}), 5.76(\mathrm{ddd}, 1 \mathrm{H}, J=17.0,10.5,8.0 \mathrm{~Hz}), 5.34-5.28(\mathrm{~m}, 2 \mathrm{H}), 4.70(\mathrm{~s})$, and 4.65 and $4.30\left(\mathrm{ABq}, J_{\mathrm{AB}}=12.0 \mathrm{~Hz}\right)(1 \mathrm{H}), 3.85-3.79(\mathrm{~m}, 1 \mathrm{H}), 3.66$ $(\mathrm{s}, 2 \mathrm{H}), 3.48(\mathrm{~s}, 1 \mathrm{H}), 2.08-2.06(\mathrm{~d}, 2 \mathrm{H}, J=7.0 \mathrm{~Hz}), 0.18(\mathrm{~s}, 9 \mathrm{H})$.

8-(2-(Benzyloxy)but-3-en-1-yl)-9-ethylidene-2-phenyloctahydro-1 $\mathrm{H}$-pyrrolo[3,4-a]indolizine-1,3(2H)-dione (23). A solution of 21 (65.5 mg, $0.13 \mathrm{mmol})$ and $\mathrm{N}$-phenylmaleimide (112 mg, 0.65 $\mathrm{mmol})$ in DCE $(3.0 \mathrm{~mL})$ was heated to reflux, and then TBAT $(70 \mathrm{mg}$, $0.13 \mathrm{mmol}$ ) was added. The mixture was refluxed for $14 \mathrm{~h}$, allowed to cool to rt, and then was concentrated under reduced pressure. The usual purification (60\% EtOAc in hexanes) afforded a mixture of diastereomers $23(14.0 \mathrm{mg}, 24 \%)$ as a pale yellow oil. ${ }^{1} \mathrm{H}$ NMR (300 $\left.\mathrm{MHz}, \mathrm{CDCl}_{3}\right) \delta(\mathrm{ppm}) 7.51-7.28(\mathrm{~m}, 10 \mathrm{H}), 5.87-5.72(\mathrm{~m})$ and 5.66 $(\mathrm{q}, J=7.0 \mathrm{~Hz})(2 \mathrm{H}), 5.31-5.24(\mathrm{~m}, 2 \mathrm{H}), 4.67$ and $4.37(\mathrm{ABq}, J=12.0$ $\mathrm{Hz})$, and 4.62 and $4.35(\mathrm{ABq}, J=12.0 \mathrm{~Hz})(2 \mathrm{H}), 3.86-3.34(\mathrm{~m}, 5 \mathrm{H})$, $3.20-2.57(\mathrm{~m}, 5 \mathrm{H}), 1.87-1.66(\mathrm{~m}, 6 \mathrm{H}) .{ }^{13} \mathrm{C} \mathrm{NMR}\left(75 \mathrm{MHz} \mathrm{CDCl}_{3}\right) \delta$ (ppm) 177.5, 177.3, 176.4, 176.3, 139.0, 138.97, 138.8, 137.1, 136.7, $132.0,131.9,129.2$, 129.19, 128.7, 128.6, 128.4, 127.9, 127.8, 127.6, $127.5,126.6,126.59,126.5,123.8,119.2,117.8,117.5,78.3,78.2,77.4$, $70.2,70.1,70.0,65.8,57.4,54.9,50.1,47.0,46.6,45.2,45.1,43.5,39.9$, 38.0, 29.9, 29.8, 29.2, 27.8, 26.9, 13.3, 13.0. IR (neat) $\nu\left(\mathrm{cm}^{-1}\right) 2919$, 2853, 1707, 1497, 1375, 1176, 729, 691. HRMS (ESI) Calcd for $\mathrm{C}_{29} \mathrm{H}_{33} \mathrm{~N}_{2} \mathrm{O}_{3}\left[\mathrm{MH}^{+}\right]$457.2486, found 457.2486.

Following the above procedure, TBAT $(71 \mathrm{mg}, 0.13 \mathrm{mmol})$ was added to a solution of $21(65.5 \mathrm{mg}, 0.13 \mathrm{mmol})$ and $N$-phenylmaleimide $(104 \mathrm{mg}, 0.60 \mathrm{mmol})$ in DCM $(3.0 \mathrm{~mL})$ at $0{ }^{\circ} \mathrm{C}$. After $20 \mathrm{~h}$ at $0{ }^{\circ} \mathrm{C}$, the mixture was concentrated under reduced pressure. The usual purification (60\% EtOAc in hexanes) afforded one diastereomer 23 $(8.80 \mathrm{mg}, 16 \%)$ as a pale yellow oil. ${ }^{1} \mathrm{H} \mathrm{NMR}\left(300 \mathrm{MHz}, \mathrm{CDCl}_{3}\right) \delta$ (ppm) 7.38-7.20 (m, 10H), 5.74-5.58 (m, 2H), 5.25-5.19 (m, 2H), 4.60 and $4.29(\mathrm{ABq}, 2 \mathrm{H}, J=12.0 \mathrm{~Hz}), 3.81-3.74(\mathrm{~m}, 1 \mathrm{H}), 3.48-3.42$ $(\mathrm{m}, 2 \mathrm{H}), 3.32-3.26(\mathrm{~m}, 2 \mathrm{H}), 3.10-2.99(\mathrm{~m}, 2 \mathrm{H}), 2.37(\mathrm{dd}, 1 \mathrm{H}, J=$ $9.5,7.0 \mathrm{~Hz}), 2.21(\mathrm{dt}, 1 \mathrm{H}, J=10.0$ and $4.5 \mathrm{~Hz}), 1.84-1.75(\mathrm{~m}, 2 \mathrm{H}), 1.72$ $(\mathrm{dd}, 3 \mathrm{H}, J=7.0,2.0 \mathrm{~Hz}), 1.63-1.56(\mathrm{~m}, 2 \mathrm{H}) .{ }^{13} \mathrm{C}$ NMR $(75 \mathrm{MHz}$, $\left.\mathrm{CDCl}_{3}\right) \delta(\mathrm{ppm}) 178.7(\mathrm{~s}), 175.1(\mathrm{~s}), 139.2(\mathrm{~d}), 139.0,135.1,134.2$, $132.1,129.0$ (d), 128.4 (d), 128.2 (d), 127.4 (d), 127.2 (d), 126.7 (d), $122.5(\mathrm{~d}), 116.7(\mathrm{t}), 77.8(\mathrm{~d}), 69.7(\mathrm{t}), 68.2(\mathrm{~d}), 56.6(\mathrm{t}), 47.7(\mathrm{~d}), 45.9$ $(\mathrm{t}), 44.2(\mathrm{~d}), 41.6(\mathrm{t}), 28.4(\mathrm{~d}), 23.7(\mathrm{t}), 12.8(\mathrm{q})$. IR (neat) $\nu\left(\mathrm{cm}^{-1}\right)$ 2933, 2859, 1711, 1498, 1383, 1194, 735, 693. HRMS (ESI) Calcd for $\mathrm{C}_{29} \mathrm{H}_{32} \mathrm{~N}_{2} \mathrm{O}_{3} \mathrm{Na}\left[\mathrm{MNa}^{+}\right] 479.2305$, found 479.2310 .

(E)-N-(3-(2-((tert-Butyldimethylsilyl)oxy)ethyl)-6-(trimethylsilyl)hex-4-en-1-yl)- $\mathrm{N}$-((trimethylsilyl)methyl)formamide (24). Following the procedure used to prepare $16,13(2.0 \mathrm{~g}, 6.95 \mathrm{mmol})$ was treated with acetic acid $(3.1 \mathrm{~mL}, 55.6 \mathrm{mmol})$ and activated zinc powder $(4.55 \mathrm{~g}, 69.5 \mathrm{mmol})$ in EtOAc $(35 \mathrm{~mL})$ and then with $\mathrm{K}_{2} \mathrm{CO}_{3}$ $(9.60 \mathrm{~g}, 69.5 \mathrm{mmol})$ and (iodomethyl)trimethylsilane $(1.4 \mathrm{~mL}, 9.04$ $\mathrm{mmol}$ ) in THF $(35 \mathrm{~mL})$, followed by the addition of $N$-formylbenzotriazole $(1.13 \mathrm{~g}, 6.95 \mathrm{mmol})$ directly to the reaction mixture. The usual purification (10\% EtOAc in hexanes) afforded a mixture of rotamers $24(2.10 \mathrm{~g}, 68 \%)$ as a pale yellow oil. ${ }^{1} \mathrm{H}$ NMR $(300 \mathrm{MHz}$, $\left.\mathrm{CDCl}_{3}\right)(\mathrm{ppm}) 7.98(\mathrm{~s})$ and $7.96(\mathrm{~s})(1 \mathrm{H}$, rotamers $), 5.38(\mathrm{dt}, 1 \mathrm{H}, J=$ $15.1,7.8 \mathrm{~Hz}), 4.91(\mathrm{dd}, 1 \mathrm{H}, J=15.1,9.1 \mathrm{~Hz}), 3.65-3.49(\mathrm{~m}, 2 \mathrm{H}), 3.17$ $(\mathrm{t}, 2 \mathrm{H}, J=7.4 \mathrm{~Hz}), 2.79\left(\mathrm{ABq}, 2 \mathrm{H}, J_{A B}=15.0 \mathrm{~Hz}\right), 2.12-1.98(\mathrm{~m}, 1 \mathrm{H})$, $1.69-1.50(\mathrm{~m}, 2 \mathrm{H}), 1.48-1.30(\mathrm{~m}, 4 \mathrm{H}), 0.88(\mathrm{~s}, 9 \mathrm{H}), 0.08(\mathrm{~s}, 9 \mathrm{H}), 0.02$ $(\mathrm{s}, 6 \mathrm{H}),-0.01(\mathrm{~s}, 9 \mathrm{H}) .{ }^{13} \mathrm{C} \mathrm{NMR}\left(75 \mathrm{MHz}, \mathrm{CDCl}_{3}\right) \delta(\mathrm{ppm}) 162.2$, $161.80,131.8,131.4,128.4,127.7,61.3,61.1,47.9,43.7,39.0,38.9,38.7$, 37., 37.2, 34.1, 34.1, 32.7, 26.1, 22.9, 22.8, 18.4, -1.2, -1.5, -1.8, -5.1. IR (neat) $\nu\left(\mathrm{cm}^{-1}\right) 2952,2928,2857,1680,1467,1432,1389,1247$, 1098, 836. HRMS (ESI) Calcd for $\mathrm{C}_{22} \mathrm{H}_{49} \mathrm{NO}_{2} \mathrm{Si}_{3} \mathrm{Na}\left[\mathrm{MNa}^{+}\right]$466.2963, found 466.2952 .

(E)-N-(3-(2-((tert-Butyldimethylsilyl)oxy)ethyl)-6-(trimethylsilyl)hex-4-en-1-yl)- $\mathrm{N}$-(cyanomethyl)formamide (25). Following the procedure used to prepare $16,13(800 \mathrm{mg}, 2.22 \mathrm{mmol})$ was treated with acetic acid $(1.00 \mathrm{~mL}, 17.8 \mathrm{mmol})$ and activated zinc powder $(1.45$ $\mathrm{g}, 22.2 \mathrm{mmol})$ in EtOAc $(10 \mathrm{~mL})$ and then with $\mathrm{K}_{2} \mathrm{CO}_{3}(769 \mathrm{mg}, 5.56$ $\mathrm{mmol})$ and bromoacetonitrile $(162 \mu \mathrm{L}, 2.33 \mathrm{mmol})$ in THF $(22 \mathrm{~mL})$ at rt for $4 \mathrm{~h}$ followed by the addition of $\mathrm{N}$-formylbenzotriazole $(360 \mathrm{mg}$, $2.45 \mathrm{mmol}$ ) directly to the reaction mixture. The latter was quenched with a solution of $1 \mathrm{~N} \mathrm{NaOH}$. The usual workup (DCM) and purification (25\% EtOAc in hexanes) afforded a mixture of rotamers $\mathbf{2 5}$ (682 mg, 77\%) as a colorless oil. ${ }^{1} \mathrm{H}$ NMR $\left(300 \mathrm{MHz}, \mathrm{CDCl}_{3}\right) \delta(\mathrm{ppm})$ $8.08(\mathrm{~s})$ and $8.04(\mathrm{~s})(1 \mathrm{H}$, rotamers $), 5.40(\mathrm{dt}, 1 \mathrm{H}, J=15.0,7.8 \mathrm{~Hz}), 4.93$ $(\mathrm{dd}, 1 \mathrm{H}, J=15.0,9.0 \mathrm{~Hz}), 4.34$ and $4.18\left(\mathrm{ABq}, 2 \mathrm{H}, J_{A B}=17.5 \mathrm{~Hz}\right), 3.67-$ $3.51(\mathrm{~m}, 2 \mathrm{H}), 3.45-3.34(\mathrm{~m}, 2 \mathrm{H}), 2.16-2.04(\mathrm{~m}, 1 \mathrm{H}), 1.79-1.68(\mathrm{~m}$, $1 \mathrm{H}), 1.63-1.39(\mathrm{~m}, 5 \mathrm{H}), 0.88(\mathrm{~s}, 9 \mathrm{H}), 0.06(\mathrm{~s}, 6 \mathrm{H}), 0.03(\mathrm{~s}, 9 \mathrm{H}) .{ }^{13} \mathrm{C}$ $\operatorname{NMR}\left(75 \mathrm{MHz}, \mathrm{CDCl}_{3}\right) \delta(\mathrm{ppm}) 162.5$ (d), 130.8 (d), $129.2(\mathrm{~d}), 114.8$ $(\mathrm{s}), 60.9(\mathrm{t}), 46.0(\mathrm{t}), 38.8(\mathrm{t}), 37.1(\mathrm{~d}), 33.6(\mathrm{t}), 30.0(\mathrm{t}), 26.1(\mathrm{q}), 23.0$ $(\mathrm{t}), 18.4(\mathrm{~s}),-1.7(\mathrm{q}),-5.1(\mathrm{q})$. IR (neat) $\nu\left(\mathrm{cm}^{-1}\right) 2953,2929,2857$, $1680,1471,1426,1247,1098,835,774$. HRMS (positive ESI) Calcd for $\mathrm{C}_{20} \mathrm{H}_{40} \mathrm{~N}_{2} \mathrm{O}_{2} \mathrm{Si}_{2} \mathrm{Na}\left[\mathrm{MNa}^{+}\right]$419.2520, found 419.2524.

(E)-N-(3-(2-Hydroxyethyl)-6-(trimethylsilyl)hex-4-en-1-yl)-N((trimethylsilyl)methyl)formamide (26). Following the procedure used to prepare $14,24(2.10 \mathrm{~g}, 4.73 \mathrm{mmol})$ was treated with camphor 
sulfonic acid (1.32 g, $5.68 \mathrm{mmol})$ in $\mathrm{MeOH}(50 \mathrm{~mL})$. The usual workup (EtOAc) and purification (40\% EtOAc in hexanes) afforded a mixture of rotamers $26(1.33 \mathrm{~g}, 85 \%)$ as a colorless oil. ${ }^{1} \mathrm{H}$ NMR $(300 \mathrm{MHz}$, $\left.\mathrm{CDCl}_{3}\right) \delta(\mathrm{ppm}) 7.96(\mathrm{~s})$ and $7.94(\mathrm{~s})(1 \mathrm{H}$, rotamers $), 5.42(\mathrm{dt}, 1 \mathrm{H}, J=$ $15.5,8.0 \mathrm{~Hz}), 4.99(\mathrm{dd}, J=15.5,9.0 \mathrm{~Hz})$ and $4.92(\mathrm{dd}, J=15.5,9.0 \mathrm{~Hz})$ (1H, rotamers), 3,68-3.54 $(2 \mathrm{H}, \mathrm{m}), 3.36-3.21(\mathrm{~m})$ and $3.17(\mathrm{t}, J=7.5$ $\mathrm{Hz})\left(2 \mathrm{H}\right.$, rotamers), 2.90 and $2.66\left(\mathrm{ABq}, J_{A B}=15.0 \mathrm{~Hz}\right)$ and $2.69(\mathrm{~s})$ (2H, rotamers), $2.11-1.99(\mathrm{~m}, 1 \mathrm{H}), 1.15$ (br. s, $1 \mathrm{H}), 1.69-1.54(\mathrm{~m}$, $2 \mathrm{H}), 1.51-1.31(\mathrm{~m}, 4 \mathrm{H}), 0.08(\mathrm{~s})$ and $0.06(\mathrm{~s})(9 \mathrm{H}$, rotamers $),-0.02(\mathrm{~s}$, $9 \mathrm{H}) .{ }^{13} \mathrm{C}$ NMR (75 MHz, $\left.\mathrm{CDCl}_{3}\right) \delta(\mathrm{ppm}) 162.4,161.8,131.8,131.2$, 128.9, 127.9, 61.0, 60.9, 47.8, 43.5, 38.7, 38.7, 38.4, 37.9, 37.5, 34.1, 34.1, 32.6, 22.9, 22.8, -1.3, -1.7, -1.9. IR (neat) $\nu\left(\mathrm{cm}^{-1}\right) 3409,2929,2894$, 2867, 1653, 1432, 1391, 1246, 843. HRMS (ESI) Calcd for $\mathrm{C}_{16} \mathrm{H}_{35} \mathrm{NO}_{2} \mathrm{Si}_{2} \mathrm{Na}\left[\mathrm{MNa}^{+}\right]$352.2098, found 352.2090.

(E)-N-(Cyanomethyl)-N-(3-(2-hydroxyethyl)-6-(trimethylsilyl)hex-4-en-1-yl)formamide (27). Following the procedure used to prepare 14,25 ( $50 \mathrm{mg}, 0.13 \mathrm{mmol}$ ) was treated with camphor sulfonic acid $(32.2 \mathrm{mg}, 0.14 \mathrm{mmol})$ in $\mathrm{MeOH}(1 \mathrm{~mL})$. The usual workup (EtOAc) and purification (80\% EtOAc in hexanes) afforded a mixture of rotamers $27(29.9 \mathrm{mg}, 84 \%)$ as a colorless oil. ${ }^{1} \mathrm{H}$ NMR $(300 \mathrm{MHz}$, $\left.\mathrm{CDCl}_{3}\right) \delta(\mathrm{ppm}) 8.08(\mathrm{~s})$ and $8.03(\mathrm{~s})(1 \mathrm{H}$, rotamers $), 5.47(\mathrm{dt}, 1 \mathrm{H}, J=$ $15.5,8.0 \mathrm{~Hz}), 4.95(\mathrm{dd}, 1 \mathrm{H}, J=15.5,9.0 \mathrm{~Hz}), 4.30$ and $4.22\left(\mathrm{ABq}, J_{A B}=\right.$ $17.5 \mathrm{~Hz})$ and $4.16(\mathrm{~s})(2 \mathrm{H}), 3.72-3.57(\mathrm{~m}, 2 \mathrm{H}), 3.40(\mathrm{dd}, 2 \mathrm{H}, J=8.0$, $6.5 \mathrm{~Hz}), 2.20-2.08(\mathrm{~m}, 1 \mathrm{H}), 1.83-1.71(\mathrm{~m}, 1 \mathrm{H}), 1.68-1.56(\mathrm{~m}, 2 \mathrm{H})$, $1.55-1.41(\mathrm{~m}, 4 \mathrm{H}), 0.00(\mathrm{~s}, 9 \mathrm{H}) .{ }^{13} \mathrm{C} \mathrm{NMR}\left(75 \mathrm{MHz}, \mathrm{CDCl}_{3}\right) \delta(\mathrm{ppm})$ $162.5(\mathrm{~d}), 130.6(\mathrm{~d}), 129.7(\mathrm{~d}), 114.9(\mathrm{~s}), 60.7(\mathrm{t}), 47.0(\mathrm{t}), 38.6(\mathrm{t}), 37.3$ (d), $33.6(\mathrm{t}), 30.2(\mathrm{t}), 23.0(\mathrm{t}),-1.7(\mathrm{q})$. IR (neat) $\nu\left(\mathrm{cm}^{-1}\right) 3425,2948$, 2885, 1667, 1430, 1397, 1177, 1055, 913. HRMS (positive ESI) Calcd for $\mathrm{C}_{14} \mathrm{H}_{26} \mathrm{~N}_{2} \mathrm{O}_{2} \mathrm{SiNa}\left[\mathrm{MNa}^{+}\right]$305.1655, found 305.1658.

(E)-N-(3-(2-Oxoethyl)-6-(trimethylsilyl)hex-4-en-1-yl)-N((trimethylsilyl)methyl)formamide (28). Following the procedure used to prepare 14, $26(919 \mathrm{mg}, 2.79 \mathrm{mmol})$ in DCM $(50 \mathrm{~mL})$ was treated with Dess-Martin periodinane $(1.42 \mathrm{~g}, 3.35 \mathrm{mmol})$ for $12 \mathrm{~h}$. The usual workup (DCM) and purification (30\% EtOAC in hexanes) afforded a mixture of rotamers $28(913 \mathrm{mg}, 94 \%)$ as a colorless oil. ${ }^{1} \mathrm{H}$ $\operatorname{NMR}\left(300 \mathrm{MHz}, \mathrm{CDCl}_{3}\right) \delta(\mathrm{ppm}) 9.67(\mathrm{t}, 1 \mathrm{H}, J=2.0 \mathrm{~Hz}), 8.00(\mathrm{~s})$ and $7.96(\mathrm{~s})(1 \mathrm{H}$, rotamers $), 5.49(\mathrm{dt}, J=16.0,8.5 \mathrm{~Hz})$ and $5.48(\mathrm{dt}, J=16.0$, $8.5 \mathrm{~Hz})(1 \mathrm{H}$, rotamers $), 5.06(\mathrm{dd}, 1 \mathrm{H}, J=15.0,8.5 \mathrm{~Hz})$ and $5.00(\mathrm{dd}, J=$ $15.0,8.5 \mathrm{~Hz})(1 \mathrm{H}$, rotamers $), 3.28(\mathrm{t}, J=7.5 \mathrm{~Hz})$ and $3.20(\mathrm{t}, J=7.5 \mathrm{~Hz})$ $(2 \mathrm{H}$, rotamers $), 2.94$ and $2.64\left(\mathrm{ABq}, J_{A B}=15.0 \mathrm{~Hz}\right)$ and $2.70(\mathrm{~s})(2 \mathrm{H}$, rotamers), $2.59-2.54(\mathrm{~m}, 1 \mathrm{H}), 2.43-2.36(\mathrm{~m}, 2 \mathrm{H}), 1.80-1.51(\mathrm{~m}, 2 \mathrm{H})$, $1.49-1.37(\mathrm{~m}, 2 \mathrm{H}), 0.09(\mathrm{~s})$ and $0.07(\mathrm{~s})(9 \mathrm{H}$, rotamers $),-0.03(\mathrm{~s}, 9 \mathrm{H})$. ${ }^{13} \mathrm{C} \mathrm{NMR}\left(75 \mathrm{MHz}, \mathrm{CDCl}_{3}\right) \delta(\mathrm{ppm}) 202.3,201.6,162.3,161.7,129.9$, 129.4, 129.0, 49.7, 49.4, 47.4, 43.3, 38.7, 35.8, 35.2, 34.0, 33.4, 32.2, 23.0, $22.9,-1.2,-1.6,-1.8,-1.9$. IR (neat) $\nu\left(\mathrm{cm}^{-1}\right) 2952,2892,1723,1663$, 1435, 1391, 1246, 1152, 841. HRMS (ESI) Calcd for $\mathrm{C}_{16} \mathrm{H}_{33} \mathrm{NO}_{2} \mathrm{Si}_{2} \mathrm{Na}$ $\left[\mathrm{MNa}^{+}\right] 350.1942$, found 350.1938 .

(E)- $N$-(Cyanomethyl)- $N$-(3-(2-oxoethyl)-6-(trimethylsilyl)hex4-en-1-yl)formamide (29). Following the procedure used to prepare 14, $27(63.0 \mathrm{mg}, 0.22 \mathrm{mmol})$ in DCM $(5 \mathrm{~mL})$ was treated with DessMartin periodinane $(114 \mathrm{mg}, 0.27 \mathrm{mmol})$ for $1 \mathrm{~h}$. The usual workup (DCM) and purification (50\% EtOAc in hexanes) afforded a mixture of rotamers $29(59.4 \mathrm{mg}, 95 \%)$ as a colorless oil. ${ }^{1} \mathrm{H}$ NMR $(8: 2)(300 \mathrm{MHz}$, $\left.\mathrm{CDCl}_{3}\right) \delta(\mathrm{ppm}) 9.70(\mathrm{t}, 1 \mathrm{H}, J=1.4 \mathrm{~Hz}), 8.10(\mathrm{~s})$ and $8.03(\mathrm{~s})(1 \mathrm{H}$, rotamers), $5.52(\mathrm{dt}, 1 \mathrm{H}, J=15.0,8.0 \mathrm{~Hz}), 5.01(\mathrm{dd}, 1 \mathrm{H}, J=15.0,8.0 \mathrm{~Hz})$, 4.42 and $4.21\left(\mathrm{ABq}, 2 \mathrm{H}, J_{A B}=17.5 \mathrm{~Hz}\right), 3.41(\mathrm{dd}, 2 \mathrm{H}, J=8.0,6.0 \mathrm{~Hz})$, $2.60-2.51(\mathrm{~m}, 1 \mathrm{H}), 2.49-2.46(\mathrm{~m}, 2 \mathrm{H}), 1.87-1.75(\mathrm{~m}, 1 \mathrm{H}), 1.54-1.47$ $(\mathrm{m}, 1 \mathrm{H}), 1.45(\mathrm{dd}, 2 \mathrm{H}, J=8.0,1.0 \mathrm{~Hz}),-0.01(\mathrm{~s}, 9 \mathrm{H}) .{ }^{13} \mathrm{C}$ NMR $(75$ $\mathrm{MHz}, \mathrm{CDCl}_{3}$ ) $\delta$ (ppm) 162.4 (d), 161.9 (d), 130.5 (d), 129.8 (d), 129.2 $(\mathrm{d}), 128.9(\mathrm{~d}), 128.2(\mathrm{~d}), 114.8(\mathrm{~s}), 49.8(\mathrm{t}), 49.4(\mathrm{t}), 45.6(\mathrm{t}), 41.6(\mathrm{t})$, $36.1(\mathrm{t}), 35.3(\mathrm{~d}), 34.7(\mathrm{~d}), 32.7(\mathrm{t}), 32.0(\mathrm{t}), 30.0(\mathrm{t}), 29.8(\mathrm{t}), 28.4(\mathrm{t})$, $23.1(\mathrm{t}),-1.6(\mathrm{q}),-1.8(\mathrm{q})$. IR (neat) $\nu\left(\mathrm{cm}^{-1}\right) 2955,2923,1719,1673$, 1421, 1394, 1243, 1178, 1151, 963, 840. HRMS (positive ESI) Calcd for $\mathrm{C}_{14} \mathrm{H}_{24} \mathrm{~N}_{2} \mathrm{O}_{2} \mathrm{SiNa}\left[\mathrm{MNa}^{+}\right]$303.1499, found 303.1503.

Methyl (E)-3-Hydroxy-2-methylene-8-(trimethylsilyl)-5-(2( $N$-((trimethylsilyl)methyl)formamido)ethyl)oct-6-enoate (30). Following the reported procedure, ${ }^{14}$ methyl acrylate $(0.2 \mathrm{~mL}, 2.29$ mmol, freshly distilled) and DABCO $(257 \mathrm{mg}, 2.29 \mathrm{mmol})$ were successively added to a solution of $28(75.0 \mathrm{mg}, 0.23 \mathrm{mmol})$ in $1,4-$ dioxane/water (1:1) (2 mL). After $72 \mathrm{~h}$ at rt, water was added. The usual workup (tert-Butyl methyl ether) and purification (30\% EtOAc in hexanes) afforded 30 as an inseparable mixture of rotamers and diastereomers $(68.4 \mathrm{mg}, 72 \%)$ as a colorless oil. ${ }^{1} \mathrm{H}$ NMR $(400 \mathrm{MHz}$, $\left.\mathrm{CDCl}_{3}\right) \delta(\mathrm{ppm}) 7.98-7.95(\mathrm{~m}, 1 \mathrm{H}), 6.23-6.21(\mathrm{~m}, 1 \mathrm{H}), 5.83-5.77$ $(\mathrm{m}, 1 \mathrm{H}), 5.51(\mathrm{dt}, J=15.5,8.0 \mathrm{~Hz})$ and $5.51(\mathrm{dt}, J=15.5,8.0 \mathrm{~Hz})$ and $5.42(\mathrm{dt}, J=15.5,8.0 \mathrm{~Hz})$ and $5.42(\mathrm{dt}, J=15.5,8.0 \mathrm{~Hz})(1 \mathrm{H}), 5.09-$ $4.98(\mathrm{~m})$ and $4.93(\mathrm{dd}, J=15.0,9.0 \mathrm{~Hz})(1 \mathrm{H}), 4.46-4.39(\mathrm{~m}, 1 \mathrm{H})$, $3.78-3.75(\mathrm{~m}, 3 \mathrm{H}), 3.21-3.11(\mathrm{~m}, 2 \mathrm{H}), 2.90$ and $2.68\left(\mathrm{ABq}, J_{A B}=15.0\right.$ $\mathrm{Hz})$ and 2.88 and $2.68\left(\mathrm{ABq}, J_{A B}=15.0 \mathrm{~Hz}\right)$ and $2.71(\mathrm{~s})(2 \mathrm{H}),(2.48-$ 2.45) $(\mathrm{m})$ and $2.32-3.34(\mathrm{~m})(1 \mathrm{H}), 1.76-1.68(\mathrm{~m}, 1 \mathrm{H}), 1.61-1.51(\mathrm{~m}$, $2 \mathrm{H}), 1.49-1.34(\mathrm{~m}, 3 \mathrm{H}), 0.09-0.07(\mathrm{~m}, 9 \mathrm{H}), 0.01-0.01(\mathrm{~m}, 9 \mathrm{H}) .{ }^{13} \mathrm{C}$ NMR $\left(75 \mathrm{MHz}, \mathrm{CDCl}_{3}\right) \delta$ (ppm) 167.1, 167.0, 162.6, 162.3, 161.8, 143.6, 143.2, 141.9, 141.8, 132.0, 131.6, 131.3, 130.6, 129.7, 129.0, 128.6, 128.3 , 125.7, 125.6, 124.8, 124.6, 70.5, 70.4, 68.9, 52.1, 52.0, 51.9, 47.9, 47.6, 43.6, 43.3, 42.4, 42.4, 41.9, 38.7, 38.6, 38.6, 37.9, 37.4, 37.4, 34.5, 34.1, 34.0, 33.4, 33.0, 32.1, 31.1, 23.0, 23.0, 22.9, -1.2, -1.7, -1.8, -1.9. IR (neat) $\nu\left(\mathrm{cm}^{-1}\right) 3392,2950,1718,1650,1436,1248,1152,842$. HRMS (ESI) Calcd for $\mathrm{C}_{20} \mathrm{H}_{39} \mathrm{NO}_{4} \mathrm{Si}_{2} \mathrm{Na}\left[\mathrm{MNa}^{+}\right]$436.2309, found 436.2307.

Methyl (E)-5-(2-(N-(Cyanomethyl)formamido)ethyl)-3-hydroxy-2-methylene-8-(trimethylsilyl)oct-6-enoate (31). Following the reported procedure, ${ }^{14} 29(1.58 \mathrm{~g}, 5.63 \mathrm{mmol})$ in 1,4-dioxane/ water $(1: 1)(6 \mathrm{~mL})$ was treated with methyl acrylate $(5.1 \mathrm{~mL}, 56.3$ $\mathrm{mmol})$ and DABCO $(6.3 \mathrm{~g}, 56.3 \mathrm{mmol})$ for $78 \mathrm{~h}$. The usual workup (tert-butyl methyl ether) and purification $\left(20 \% \mathrm{Et}_{2} \mathrm{O}\right.$ in DCM) afforded as an inseparable mixture of rotamers and diastereomers $31(1.99 \mathrm{~g}$, $91 \%)$ as a yellow oil. ${ }^{1} \mathrm{H} \mathrm{NMR}\left(300 \mathrm{MHz}, \mathrm{CDCl}_{3}\right) \delta(\mathrm{ppm}) 8.10(\mathrm{~s})$, and $8.08(\mathrm{~s})$, and $8.05(\mathrm{~s})(1 \mathrm{H}), 6.24(\mathrm{~s})$, and $6.22(\mathrm{~s})(1 \mathrm{H}), 5.81(\mathrm{~s}, 1 \mathrm{H})$, $5.55(\mathrm{dt}, J=15.5,8.0 \mathrm{~Hz})$, and $5.46(\mathrm{dt}, J=15.5,8.0 \mathrm{~Hz})(1 \mathrm{H}), 5.08-$ $5.02(\mathrm{~m})$, and $4.96(\mathrm{dd}, J=15.5,9.0 \mathrm{~Hz})(1 \mathrm{H}), 4.48-4.41(\mathrm{~m}, 1 \mathrm{H}), 4.33$ and $4.20\left(\mathrm{ABq}, J_{A B}=17.5 \mathrm{~Hz}\right)$ and $4.27(\mathrm{~s})(2 \mathrm{H}), 3.78(\mathrm{~s})$ and $3.77(\mathrm{~s})$ $(3 \mathrm{H}), 3.44-3.38(2 \mathrm{H}), 2.39-2.31(\mathrm{~m}, 1 \mathrm{H}), 1.72-1.45(\mathrm{~m}, 7 \mathrm{H}), 0.02$ (s) and $0.01(\mathrm{~s})(9 \mathrm{H}) .{ }^{13} \mathrm{C} \mathrm{NMR}\left(75 \mathrm{MHz}, \mathrm{CDCl}_{3}\right) \delta(\mathrm{ppm}) 166.9(\mathrm{~s})$, 162.3 (d), 143.0 (s), 130.9 (d), 130.3 (d), 129.9 (d), 129.4 (d), 125.5 $(\mathrm{t}), 124.7(\mathrm{t}), 114.7(\mathrm{~s}), 69.9(\mathrm{~d}), 68.8(\mathrm{~d}), 52.0(\mathrm{q}), 51.9(\mathrm{q}), 45.9(\mathrm{t})$, $45.8(\mathrm{t}), 42.2(\mathrm{t}), 37.3(\mathrm{~d}), 37.2(\mathrm{~d}), 34.0(\mathrm{t}), 32.5(\mathrm{t}), 30.0(\mathrm{t}), 23.0(\mathrm{t})$, $22.9(\mathrm{t}),-1.8$ (q). IR (neat) $\nu\left(\mathrm{cm}^{-1}\right) 3471,2952,1716,1671,1434$, 1246, 1152, 849 HRMS (positive ESI) Calcd for $\mathrm{C}_{18} \mathrm{H}_{30} \mathrm{~N}_{2} \mathrm{O}_{4} \mathrm{SiNa}$ $\left[\mathrm{MNa}^{+}\right]$389.1867, found 389.1871.

Methyl (E)-3-(Benzyloxy)-2-methylene-8-(trimethylsilyl)-5(2-(N-((trimethylsilyl)methyl)formamido)ethyl)oct-6-enoate (32). Following the procedure used to prepare 19, $30(312 \mathrm{mg}, 0.75$ $\mathrm{mmol})$ in $\mathrm{THF}(8 \mathrm{~mL})$ at $0{ }^{\circ} \mathrm{C}$ was treated with $\mathrm{NaH}(60 \%$ dispersion in mineral oil, $60.0 \mathrm{mg}, 1.51 \mathrm{mmol})$ and then with $n-\mathrm{Bu}_{4} \mathrm{NI}(279 \mathrm{mg}, 0.75$ $\mathrm{mmol})$ and benzyl bromide $(0.90 \mathrm{~mL}, 7.54 \mathrm{mmol})$ for $16 \mathrm{~h}$. The usual workup $\left(\mathrm{Et}_{2} \mathrm{O}\right)$ and purification (15\% EtOAc in hexanes) afforded a mixture of rotamers and diastereomers $32(240 \mathrm{mg}, 63 \%)$ as a colorless oil. ${ }^{1} \mathrm{H}$ NMR (400 MHz, $\left.\mathrm{CDCl}_{3}\right) \delta(\mathrm{ppm}) 7.99-7.95(\mathrm{~m}, 1 \mathrm{H}), 7.41-$ $7.32(\mathrm{~m}, 5 \mathrm{H}), 6.38-6.35(\mathrm{~m}, 1 \mathrm{H}), 5.96-5.94(\mathrm{~m}, 1 \mathrm{H}), 5.45-5.21(\mathrm{~m}$, $1 \mathrm{H}), 5.08-4.86(\mathrm{~m}, 1 \mathrm{H}), 4.51$ and $4.25(\mathrm{ABq}, J=11.5 \mathrm{~Hz})$, and 4.47 and $4.23(\mathrm{ABq}, J=11.5 \mathrm{~Hz})(2 \mathrm{H}), 4.38-4.28(\mathrm{~m}, 1 \mathrm{H}), 3.77-3.77(\mathrm{~m}, 3 \mathrm{H})$, 3.29-3.19 (m), and 3.18-3.11 (m), and 3.10-3.00 (m) $(2 \mathrm{H}), 2.88(\mathrm{~d}, J$ $=15.0 \mathrm{~Hz})$, and $2.85(\mathrm{~d}, J=15.0 \mathrm{~Hz})$, and $2.68(\mathrm{~s})$, and $2.65(\mathrm{~d}, J=26.0$ $\mathrm{Hz})$, and $2.61(\mathrm{~d}, J=26 \mathrm{~Hz}), 1.69-1.29(\mathrm{~m}, 6 \mathrm{H}), 0.08(\mathrm{~s})$, and $0.06(\mathrm{~s})$, and $0.05(\mathrm{~s})(9 \mathrm{H}), 0.00(\mathrm{~s})$ and $-0.08(\mathrm{~s})(9 \mathrm{H}) .{ }^{13} \mathrm{C}$ NMR $(75 \mathrm{MHz}$, $\left.\mathrm{CDCl}_{3}\right) \delta(\mathrm{ppm}) 166.8(\mathrm{~s}), 162.2(\mathrm{~d}), 161.7(\mathrm{~d}), 141.7(\mathrm{~s}), 141.5(\mathrm{~s})$, $141.0(\mathrm{~s}), 138.5(\mathrm{~s}), 138.2(\mathrm{~s}), 138.2(\mathrm{~s}), 131.6(\mathrm{~d}), 131.4(\mathrm{~d}), 131.1$ (d), 129.3 (d), 128.7 (d), 128.6 (d), 128.5 (d), 128.4 (d), 128.2 (d), 128.1 (d), $128.0(\mathrm{~d}), 127.9(\mathrm{~d}), 127.8(\mathrm{~d}), 127.7(\mathrm{~d}), 125.7(\mathrm{t}), 125.1(\mathrm{t}), 75.7$ (d), 75.5(d), 75.4(d), 71.4(t), 71.1 (t), 52.0 (q), 51.8 (q), 48.0(t), 47.7 $(\mathrm{t}), 43.7(\mathrm{t}), 43.6(\mathrm{t}), 43.2(\mathrm{t}), 43.0(\mathrm{t}), 42.3(\mathrm{t}), 38.7(\mathrm{t}), 38.6(\mathrm{t}), 38.3$ $(\mathrm{d}), 37.7(\mathrm{~d}), 37.0(\mathrm{~d}), 34.6(\mathrm{t}), 34.1(\mathrm{t}), 34.1(\mathrm{t}), 33.0(\mathrm{t}), 32.6(\mathrm{t}), 23.0$ $(\mathrm{t}), 22.9(\mathrm{t}),-1.2(\mathrm{q}),-1.5(\mathrm{q}),-1.6(\mathrm{q}),-1.7(\mathrm{q}),-1.9(\mathrm{q})$. IR (neat) $\nu\left(\mathrm{cm}^{-1}\right) 2950,2888,1718,1667,1519,1496,1248,1192,1150,1077$, 843. HRMS (positive ESI) Calcd for $\mathrm{C}_{27} \mathrm{H}_{45} \mathrm{NO}_{4} \mathrm{Si}_{2} \mathrm{Na}\left[\mathrm{MNa}{ }^{+}\right]$ 526.2779 , found 526.2775 .

Methyl (E)-5-(2-(N-(Cyanomethyl)formamido)ethyl)-2-methylene-3-((triethylsilyl)oxy)-8-(trimethylsilyl)oct-6-enoate (33). Triethysilyl trifluoromethanesulfonate $(65 \mu \mathrm{L}, 0.29 \mathrm{mmol})$ was added dropwise to a solution of $31(88.0 \mathrm{mg}, 0.24 \mathrm{mmol})$ and triethylamine 
$(50 \mu \mathrm{L}, 0.36 \mathrm{mmol})$ in $\mathrm{DCM}(2.5 \mathrm{~mL})$ at $0{ }^{\circ} \mathrm{C}$. The mixture was stirred at $\mathrm{rt}$ for $1 \mathrm{~h} 30$. Then, water was added. The usual workup (DCM) and purification (30\% EtOAc in hexanes) afforded a mixture of rotamers and diastereomers 33 (107 mg, 93\%) as a colorless oil. ${ }^{1} \mathrm{H}$ NMR $(300 \mathrm{MHz}$, $\left.\mathrm{CDCl}_{3}\right) \delta(\mathrm{ppm}) 8.03(\mathrm{~s})$ and $8.02(\mathrm{~s})(1 \mathrm{H}), 6.22(\mathrm{~s}, 1 \mathrm{H}), 5.91(\mathrm{~s})$ and $5.87(\mathrm{~s})(1 \mathrm{H}), 5.47(\mathrm{dt}, J=15.5,8.0 \mathrm{~Hz})$ and $5.35(\mathrm{dt}, J=15.5,8.0 \mathrm{~Hz})$ $(1 \mathrm{H}), 5.01(\mathrm{dd}, J=15.5,9.0 \mathrm{~Hz})$ and $5.07-4.95(\mathrm{~m})(1 \mathrm{H}), 4.62-5.46$ $(\mathrm{m}, 1 \mathrm{H}), 4.36$ and $4.15\left(\mathrm{ABq}, J_{A B}=17.5 \mathrm{~Hz}\right)$, and 4.33 and $4.20(\mathrm{ABq}$, $\left.J_{A B}=17.5 \mathrm{~Hz}\right)(2 \mathrm{H}), 3.74(\mathrm{~s}, 3 \mathrm{H}), 3.42-3.31(\mathrm{~m}, 2 \mathrm{H}), 2.23-2.05(\mathrm{~m}$, $1 \mathrm{H}), 1.90-1.37(\mathrm{~m}, 6 \mathrm{H}), 0.96-0.89(\mathrm{~m}, 9 \mathrm{H}), 0.61-0.52(\mathrm{~m}, 6 \mathrm{H}), 0.02$ (s) and $0.01(\mathrm{~s})(9 \mathrm{H}) .{ }^{13} \mathrm{C} \mathrm{NMR}\left(76 \mathrm{MHz}, \mathrm{CDCl}_{3}\right) \delta(\mathrm{ppm}) 166.7(\mathrm{~s})$, $166.6(\mathrm{~s}), 162.4$ (d), 162.3 (d), $144.9(\mathrm{~s}), 143.7$ (s), $131.3(\mathrm{~d}), 131.1$ (d), $129.2(\mathrm{~d}), 128.3(\mathrm{~d}), 124.9(\mathrm{t}), 124.7(\mathrm{t}), 124.6(\mathrm{t}), 114.8(\mathrm{~s}), 69.0(\mathrm{~d})$, $68.6(\mathrm{~d}), 51.8(\mathrm{q}), 51.9(\mathrm{q}), 45.8(\mathrm{t}), 45.7(\mathrm{t}), 45.2(\mathrm{t}), 43.9(\mathrm{t}), 37.5(\mathrm{~d})$, $36.8(\mathrm{~d}), 36.5(\mathrm{~d}), 36.1(\mathrm{t}), 34.4(\mathrm{t}), 33.2(\mathrm{t}), 33.0(\mathrm{t}), 30.1(\mathrm{t}), 30.0(\mathrm{t})$, $23.2(\mathrm{t}), 22.9(\mathrm{t}), 7.0(\mathrm{q}), 6.9(\mathrm{q}), 5.1(\mathrm{~d}), 4.9(\mathrm{~d}),-1.7(\mathrm{q}),-1.8(\mathrm{q})$. IR (neat) $\nu\left(\mathrm{cm}^{-1}\right) 2953,2913,2877,1718,1681,1436,1247,1152,1088$, 849. HRMS (positive ESI) Calcd for $\mathrm{C}_{24} \mathrm{H}_{44} \mathrm{~N}_{2} \mathrm{O}_{4} \mathrm{Si}_{2} \mathrm{Na}$ [ $\left.\mathrm{MNa}^{+}\right]$ 503.2732, found 503.2741.

4-(2-(Benzyloxy)-3-(methoxycarbonyl)but-3-en-1-yl)-1((trimethylsilyl)methyl)-5-vinyl-2,3,4,5-tetrahydropyridin-1ium Trifluoromethanesulfonate (34) and 4-(2-(Benzyloxy)-3(methoxycarbonyl)but-3-en-1-yl)-5-ethylidene-1-((trimethylsilyl)methyl)-2,3,4,5-tetrahydropyridin-1-ium trifluoromethanesulfonate (35). $\mathrm{Tf}_{2} \mathrm{O}(32 \mu \mathrm{L}, 190 \mu \mathrm{mol})$ was added to a solution of $33(47.6 \mathrm{mg}, 94 \mu \mathrm{mol})$ and DTBMP $(21.3 \mathrm{mg}, 100 \mu \mathrm{mol})$ in DCM $(1.0 \mathrm{~mL})$ at $-78^{\circ} \mathrm{C}$. After $20 \mathrm{~min}$, the mixture was allowed to warm up at $\mathrm{rt}$ for $30 \mathrm{~min} .{ }^{1} \mathrm{H}$ NMR analysis of an aliquot showed the formation of the iminium ion 34: ${ }^{1} \mathrm{H}$ NMR $\left(300 \mathrm{MHz}, \mathrm{CD}_{2} \mathrm{Cl}_{2}\right)$ characteristic signals $\delta(\mathrm{ppm}) 8.23(\mathrm{~s})$ and $8.22(\mathrm{~s})(1 \mathrm{H}), 7.39-7.30(\mathrm{~m}$, $5 \mathrm{H}), 6.39(\mathrm{~s})$ and $6.38(\mathrm{~s})(1 \mathrm{H}), 6.00(\mathrm{~s})$ and $5.96(\mathrm{~s})(1 \mathrm{H}), 5.74-5.60$ $(\mathrm{m}, 1 \mathrm{H}), 5.47-5.36(\mathrm{~m}, 2 \mathrm{H}), 4.58$ and $4.21(\mathrm{ABq}, J=11.5 \mathrm{~Hz})$, and 4.50 and $4.27(\mathrm{ABq}, J=11.5 \mathrm{~Hz})(2 \mathrm{H}), 4.42(\mathrm{~d}, J=3.0 \mathrm{~Hz})$, and $4.40(\mathrm{~d}, J=$ $3.0 \mathrm{~Hz})$, and $5.34(\mathrm{~s})$ and $4.32(\mathrm{~s})(1 \mathrm{H}), 3.76(\mathrm{~s})$ and $3.75(\mathrm{~s})(3 \mathrm{H})$, $1.88-1.78(\mathrm{~m}, 2 \mathrm{H}), 0.21(\mathrm{~s})$ and $0.20(\mathrm{~s})(9 \mathrm{H})$. The reaction mixture was concentrated. The usual purification ( 0 to $10 \% \mathrm{MeOH}$ in EtOAc) afforded 35 as brown yellow oil. ${ }^{1} \mathrm{H}$ NMR $\left(300 \mathrm{MHz}, \mathrm{CDCl}_{3}\right)$ characteristic signals $\delta(\mathrm{ppm}) 8.38(\mathrm{~s}, 1 \mathrm{H}), 7.37-7.26(\mathrm{~m}, 5 \mathrm{H}), 7.07-$ $6.97(\mathrm{~m}, 1 \mathrm{H}), 6.42(\mathrm{~s}, 1 \mathrm{H}), 6.05(\mathrm{~s})$ and $6.00(\mathrm{~s})(1 \mathrm{H}), 4.64$ and 4.26 $(\mathrm{ABq}, J=11.5 \mathrm{~Hz})$, and 4.54 and $4.24(\mathrm{ABq}, J=11.5 \mathrm{~Hz})(2 \mathrm{H}), 4.35-$ $4.30(\mathrm{~m}, 1 \mathrm{H}), 3.67-3.41(\mathrm{~m}, 4 \mathrm{H}), 0.17(\mathrm{~s}, 9 \mathrm{H})$.

1-(Cyanomethyl)-5-ethylidene-4-(3-(methoxycarbonyl)-2((triethylsilyl)oxy)but-3-en-1-yl)-2,3,4,5-tetrahydropyridin-1ium Trifluoromethanesulfonate (39). $\mathrm{Tf}_{2} \mathrm{O}(3.7 \mu \mathrm{L}, 21.7 \mu \mathrm{mol})$ was added to $33(9.5 \mathrm{mg}, 19.8 \mu \mathrm{mol})$ and DTBMP $(4.5 \mathrm{mg}, 21.7 \mu \mathrm{mol})$ in $\mathrm{CD}_{2} \mathrm{Cl}_{2}(0.6 \mathrm{~mL})$ at $0{ }^{\circ} \mathrm{C}$ in an NMR tube. The ${ }^{1} \mathrm{H}$ NMR spectrum of 39 was recorded after $15 \mathrm{~min}$ at rt. ${ }^{1} \mathrm{H}$ NMR $\left(300 \mathrm{MHz}, \mathrm{CD}_{2} \mathrm{Cl}_{2}\right)$ characteristic signals $\delta(\mathrm{ppm}) 8.84(\mathrm{~s})$, and $8.82(\mathrm{~s})$, and $8.76-8.52(\mathrm{~m})$ $(1 \mathrm{H}), 7.35(\mathrm{q}, J=7.5 \mathrm{~Hz})$, and $7.28-7.18(\mathrm{~m})(1 \mathrm{H}), 6.32(\mathrm{~s})$, and $6.27-$ $6.20(\mathrm{~m})(1 \mathrm{H}), 6.02(\mathrm{~s})$, and $6.00-5.86(\mathrm{~m})(1 \mathrm{H}), 5.15(\mathrm{~s}, 1 \mathrm{H}), 4.80-$ $4.68(\mathrm{~m}, 1 \mathrm{H}), 3.91-3.86(\mathrm{~m}, 1 \mathrm{H}), 3.76(\mathrm{~s})$, and $3.75(\mathrm{~s})$, and 3.74-7.72 $(\mathrm{m})(3 \mathrm{H}), 2.23(\mathrm{~d}, J=7.0 \mathrm{~Hz})$, and $2.17(\mathrm{~d}, J=7.0 \mathrm{~Hz})(1 \mathrm{H}), 0.99-0.90$ $(\mathrm{m}, 9 \mathrm{H}), 0.67-0.58(\mathrm{~m}, 6 \mathrm{H})$.

Methyl 2-Cyano-4-((triethylsilyl)oxy)-7-vinyloctahydro-1,6ethanoindole-3a-carboxylate (41). $\mathrm{Tf}_{2} \mathrm{O}(20 \mu \mathrm{L}, 0.14 \mathrm{mmol})$ was added to a solution of $33(49.8 \mathrm{mg}, 0.10 \mathrm{mmol})$ and DTBMP $(23.4 \mathrm{mg}$, $0.14 \mathrm{mmol})$ in $\mathrm{DCM}(1.0 \mathrm{~mL})$ at $0{ }^{\circ} \mathrm{C}$. After $15 \mathrm{~min}, i-\mathrm{Pr}_{2} \mathrm{NEt}(108 \mu \mathrm{L}$, $0.62 \mathrm{mmol}$ ) was added at $0^{\circ} \mathrm{C}$. The mixture was then stirred for $3 \mathrm{~h}$ at rt. Saturated aqueous $\mathrm{NaHCO}_{3}$ was added. The usual workup (DCM) and purification (70\% EtOAc in hexanes) afforded 41 in traces. ${ }^{1} \mathrm{H}$ NMR $\left(300 \mathrm{MHz}, \mathrm{CDCl}_{3}\right)$ characteristic signals $\delta(\mathrm{ppm}) 5.77-5.65(\mathrm{~m}, 1 \mathrm{H})$, $5.33-5.23(\mathrm{~m}, 2 \mathrm{H}), 4.68(\mathrm{t}, 1 \mathrm{H}, J=6.0 \mathrm{~Hz}), 3.87(\mathrm{~d}, 1 \mathrm{H}, J=4.0 \mathrm{~Hz})$, $2.91-2.85(\mathrm{~m}, 1 \mathrm{H}), 2.50(\mathrm{td}, 1 \mathrm{H}, J=12.0,2.5 \mathrm{~Hz}), 2.29-2.20(\mathrm{~m}, 1 \mathrm{H})$, $2.03-1.98(\mathrm{~m}, 1 \mathrm{H}), 1.90-1.83(\mathrm{~m}, 1 \mathrm{H}), 1.37-1.25(\mathrm{~m}, 4 \mathrm{H}), 0.96-0.91$ (m, 9H), 0.62-0.54 (m, 6H). HRMS (positive ESI) Calcd for $\mathrm{C}_{21} \mathrm{H}_{34} \mathrm{~N}_{2} \mathrm{O}_{3} \mathrm{SiNa}\left[\mathrm{MNa}^{+}\right]$413.2231, found 413.2223.

Methyl 2-Cyano-4-hydroxy-7-vinyloctahydro-1,6-ethanoindole-3a-carboxylate. Following the procedure used to prepare 41, $i$ - $\mathrm{Pr}_{2} \mathrm{NEt}$ was added at $80{ }^{\circ} \mathrm{C}$ in DCE to afford the corresponding alcohol in traces. ${ }^{1} \mathrm{H}$ NMR $\left(300 \mathrm{MHz}, \mathrm{CDCl}_{3}\right)$ characteristic signals $\delta$ (ppm) $5.74-5.65(\mathrm{~m}, 1 \mathrm{H}), 5.33-5.23(\mathrm{~m}, 2 \mathrm{H}), 4.46(\mathrm{dd}, 1 \mathrm{H}, J=7.6,5.9$
$\mathrm{Hz}, 1 \mathrm{H}), 3.88(\mathrm{~d}, 1 \mathrm{H}, J=4.5 \mathrm{~Hz}), 2.95-2.85(\mathrm{~m}, 1 \mathrm{H}), 2.55(\mathrm{td}, 1 \mathrm{H}, J=$ 12.0, $3.0 \mathrm{~Hz}$ ), 2.34 (ddd, $1 \mathrm{H}, J=10.5,9.5,4.4 \mathrm{~Hz}), 2.08-2.03(\mathrm{~m}, 1 \mathrm{H})$, 1.88 (ddd, $1 \mathrm{H}, J=14.0,5.7,3.6 \mathrm{~Hz}$ ). HRMS (positive ESI) Calcd for $\mathrm{C}_{15} \mathrm{H}_{20} \mathrm{~N}_{2} \mathrm{O}_{3} \mathrm{Na}\left[\mathrm{MNa}^{+}\right]$299.1366, found 299.1367.

tert-Butyl((5-methoxy-3-(2-nitroethyl)pent-4-en-1-yl)oxy)dimethylsilane (42). A solution of $n-\operatorname{BuLi}(2.5 \mathrm{M}$ in hexane, $30.5 \mathrm{~mL}$, $76.2 \mathrm{mmol}$ ) was added dropwise to a solution of methoxymethyltriphenylphosphonium chloride $(26.1 \mathrm{~g}, 76.2 \mathrm{mmol})$ in THF $(250 \mathrm{~mL})$ at $0{ }^{\circ} \mathrm{C}$. The solution was stirred for $30 \mathrm{~min}$ at $0{ }^{\circ} \mathrm{C}$, and then a solution of $10(7.00 \mathrm{~g}, 25.4 \mathrm{mmol})$ in THF $(20 \mathrm{~mL})$ was added. The solution was stirred for $7 \mathrm{~h}$ at rt. A buffer solution of acetic acid $\mathrm{pH}=4.7$ was added. The two layers were separated. The usual workup (EtOAc) and purification ( 1 to $2 \%$ EtOAc in hexanes) gave a hardly separable 3:1 mixture of $E / Z$-isomers $\mathbf{4 2}(4.19 \mathrm{mg}, 55 \%)$ as a colorless oil. $(Z)-42$ (minor): ${ }^{1} \mathrm{H} \mathrm{NMR}\left(300 \mathrm{MHz}, \mathrm{CDCl}_{3}\right) \delta(\mathrm{ppm}) 5.98(\mathrm{~d}, 1 \mathrm{H}, J=6.0 \mathrm{~Hz})$, $4.39-4.34(\mathrm{~m}, 2 \mathrm{H}), 4.04(\mathrm{dd}, 1 \mathrm{H}, J=9.5,6.0 \mathrm{~Hz}), 3.63-3.51(\mathrm{~m}, 2 \mathrm{H})$, 3.55 (s. 3H), 2.76-2.64 (m, 1H), 2.25-2.13 (m, 1H), 1.86-1.73 (m, $1 \mathrm{H}), 1.68-1.59(\mathrm{~m}, 1 \mathrm{H}), 1.52-1.41(\mathrm{~m}, 1 \mathrm{H}), 0.88(\mathrm{~s}, 9 \mathrm{H}), 0.03(\mathrm{~s}, 6 \mathrm{H})$. ${ }^{13} \mathrm{C}$ NMR $\left(75 \mathrm{MHz}, \mathrm{CDCl}_{3}\right) \delta(\mathrm{ppm}) 148.2(\mathrm{~d}), 108.1(\mathrm{~d}), 74.3(\mathrm{t})$, $61.2(\mathrm{t}), 59.7(\mathrm{q}), 38.9(\mathrm{t}), 33.2(\mathrm{t}), 28.8(\mathrm{~d}), 26.1(\mathrm{q}), 18.4(\mathrm{~s}),-5.2(\mathrm{q})$. IR (neat) $\nu\left(\mathrm{cm}^{-1}\right) 2929,2856,1662,1551,1254,1097,834$. HRMS (positive ESI) Calcd for $\mathrm{C}_{14} \mathrm{H}_{29} \mathrm{NO}_{4} \mathrm{SiNa}\left[\mathrm{MNa}^{+}\right] 326.1758$, found 326.1742. (E)-42 (major): ${ }^{1} \mathrm{H}$ NMR (300 MHz, $\left.\mathrm{CDCl}_{3}\right) \delta$ (ppm) 6.28 $(\mathrm{d}, 1 \mathrm{H}, J=13.0 \mathrm{~Hz}), 4.41-4.36(\mathrm{~m}, 3 \mathrm{H}), 3.68-3.55(\mathrm{~m}, 2 \mathrm{H}), 3.52(\mathrm{~s}$. $3 \mathrm{H}), 2.24-2.10(\mathrm{~m}, 2 \mathrm{H}), 1.89-1.76(\mathrm{~m}, 1 \mathrm{H}), 1.71-1.60(\mathrm{~m}, 1 \mathrm{H})$, $1.50-1.39(\mathrm{~m}, 1 \mathrm{H}), 0.88(\mathrm{~s}, 9 \mathrm{H}), 0.03(\mathrm{~s}, 6 \mathrm{H}) .{ }^{13} \mathrm{C} \mathrm{NMR}(75 \mathrm{MHz}$, $\left.\mathrm{CDCl}_{3}\right) \delta \mathrm{ppm} 149.1(\mathrm{~d}), 104.2(\mathrm{~d}), 74.2(\mathrm{t}), 60.5(\mathrm{t}), 56.3(\mathrm{q}), 39.1(\mathrm{t})$, $33.6(\mathrm{t}), 32.9(\mathrm{~d}), 26.1(\mathrm{q}), 18.4(\mathrm{~s}),-5.2(\mathrm{q})$. IR (neat) $\nu\left(\mathrm{cm}^{-1}\right) 2929$, 2857, 1651, 1551, 1252, 1102, 834. HRMS (positive ESI) Calcd for $\mathrm{C}_{14} \mathrm{H}_{29} \mathrm{NO}_{4} \mathrm{SiNa}\left[\mathrm{MNa}^{+}\right] 326.1758$, found 326.1731 .

(E)-N-(3-(2-((tert-Butyldimethylsilyl)oxy)ethyl)-5-methoxypent-4-en-1-yl)- $N$-(cyanomethyl)formamide (43). Following the procedure used to prepare $16,42(2.69 \mathrm{~g}, 8.86 \mathrm{mmol})$ was treated with acetic acid $(7.4 \mathrm{~mL}, 133 \mathrm{mmol})$ and activated zinc powder $(8.70 \mathrm{~g}, 133$ $\mathrm{mmol})$ in EtOAc $(40 \mathrm{~mL})$ and then with $\mathrm{K}_{2} \mathrm{CO}_{3}(6.13 \mathrm{~g}, 44.3 \mathrm{mmol})$ and bromoacetonitrile $(930 \mu \mathrm{L}, 13.3 \mathrm{mmol})$ in THF $(90 \mathrm{~mL})$, followed by the addition of $N$-formylbenzotriazole $(1.43 \mathrm{~g}, 9.75 \mathrm{mmol})$ directly to the reaction mixture. The usual workup (DCM) and purification (30\% EtOAc in hexanes) afforded a mixture of rotamers $43(2.30 \mathrm{~g}, 76 \%)$ as a colorless oil. ${ }^{1} \mathrm{H}$ NMR $\left(300 \mathrm{MHz}, \mathrm{CDCl}_{3}\right) \delta(\mathrm{ppm}) 8.08(\mathrm{~s})$ and $(8.06)$ (s) $(1 \mathrm{H}$, rotamers $), 6.28(\mathrm{~d}, J=12.5 \mathrm{~Hz})$ and $6.26(\mathrm{~d}, J=12.5 \mathrm{~Hz})(1 \mathrm{H}$, rotamers), $4.42-4.15(\mathrm{~m}, 3 \mathrm{H}), 3.67-3.55(\mathrm{~m}, 2 \mathrm{H}), 3.52(\mathrm{~s} .3 \mathrm{H}), 3.44-$ $3.38(\mathrm{~m}, 2 \mathrm{H}), 2.14-2.01(\mathrm{~m}, 1 \mathrm{H}), 1.84-1.72(\mathrm{~m}, 1 \mathrm{H}), 1.68-1.57(\mathrm{~m}$, $1 \mathrm{H}), 1.53-1.36(\mathrm{~m}, 2 \mathrm{H}), 0.88(\mathrm{~s}, 9 \mathrm{H}), 0.03(\mathrm{~s}, 6 \mathrm{H}) .{ }^{13} \mathrm{C}$ NMR $(75$ $\left.\mathrm{MHz}, \mathrm{CDCl}_{3}\right) \delta$ (ppm) 162.4 (d), 161.8 (d, rotamer), 148.7 (d), 148.4 $(\mathrm{d}$, rotamer $), 114.8(\mathrm{~s}), 105.2(\mathrm{~d}$, rotamer $), 104.9(\mathrm{~d}), 60.6(\mathrm{t}$, rotamer $)$, $60.4(\mathrm{t}) 56.3(\mathrm{q}), 56.2(\mathrm{q}$, rotamer $), 46.0(\mathrm{t}), 42.1(\mathrm{t}$, rotamer $), 39.2(\mathrm{t})$, $39.1(\mathrm{t}$, rotamer $), 36.1(\mathrm{t}$, rotamer $), 34.1(\mathrm{t}), 33.0(\mathrm{~d}$, rotamer $), 32.4(\mathrm{~d})$, $30.1(\mathrm{t}), 26.0$ (q), $18.3(\mathrm{~s}),-5.2$ (q). IR (neat) $\nu\left(\mathrm{cm}^{-1}\right)$ 2929, 2855, 1676, 1651, 1250, 1086, 834. HRMS (positive ESI) Calcd for $\mathrm{C}_{17} \mathrm{H}_{32} \mathrm{~N}_{2} \mathrm{O}_{3} \mathrm{SiNa}\left[\mathrm{MNa}^{+}\right]$363.2074, found 363.2077.

(E)-N-(Cyanomethyl)-N-(5-methoxy-3-(2-oxoethyl)pent-4en-1-yl)formamide (44). A solution of TBAF (1.0 M in THF, $7.4 \mathrm{~mL}$, $7.4 \mathrm{mmol})$ was added to a solution of $43(2.30 \mathrm{mg}, 6.75 \mathrm{mmol})$ in THF $(140 \mathrm{~mL})$. After $6 \mathrm{~h}$, saturated aqueous $\mathrm{NaCl}$ was added. The usual workup $\left(\mathrm{Et}_{2} \mathrm{O}\right)$ afforded crude alcohol that was used directly in the next step. For characterization purposes, a small portion was purified using usual conditions ( $2 \% \mathrm{MeOH}$ in EtOAc) to afford a mixture of rotamers of the corresponding alcohol as a pale yellow oil. ${ }^{1} \mathrm{H}$ NMR $(300 \mathrm{MHz}$, $\left.\mathrm{CDCl}_{3}\right) \delta(\mathrm{ppm}) 8.08(\mathrm{~s})$ and $8.04(\mathrm{~s})(1 \mathrm{H}$, rotamers $), 6.32(\mathrm{~d}, J=12.5$ $\mathrm{Hz})$ and $6.30(\mathrm{~d}, J=12.5 \mathrm{~Hz})(1 \mathrm{H}$, rotamers $), 4.39(\mathrm{dd}, 1 \mathrm{H}, J=12.5$, $10.0 \mathrm{~Hz}), 4.25(\mathrm{ABq}, J=17.0 \mathrm{~Hz})$ and $4.17(\mathrm{~s})(2 \mathrm{H}$, rotamers $), 3.71-$ $3.57(\mathrm{~m}, 2 \mathrm{H}), 3.52(\mathrm{~s} .3 \mathrm{H}), 3.41(\mathrm{dd}, 2 \mathrm{H}, J=8.0,6.5 \mathrm{~Hz}), 2.16-2.03(\mathrm{~m}$, $1 \mathrm{H}), 1.86-1.60(\mathrm{~m}, 3 \mathrm{H}), 1.54-1.41(\mathrm{~m}, 2 \mathrm{H}) .{ }^{13} \mathrm{C}$ NMR $(75 \mathrm{MHz}$, $\left.\mathrm{CDCl}_{3}\right) \delta(\mathrm{ppm}) 162.6(\mathrm{~d}), 162.0(\mathrm{~d}$, rotamer), $148.8(\mathrm{~d}), 148.5(\mathrm{~d}$, rotamer $), 115.0(\mathrm{~s}), 105.2(\mathrm{~d}$, rotamer $), 104.9(\mathrm{~d}), 60.5(\mathrm{t}$, rotamer $)$, $60.3(\mathrm{t}) 56.4(\mathrm{q}), 56.3(\mathrm{q}$, rotamer $), 46.1(\mathrm{t}), 42.1(\mathrm{t}$, rotamer $), 39.0(\mathrm{t})$, $38.8(\mathrm{t}$, rotamer $), 36.2(\mathrm{t}$, rotamer $), 34.0(\mathrm{t}), 33.2(\mathrm{~d}$, rotamer $), 33.1(\mathrm{t}$, rotamer), $32.6(\mathrm{~d}), 30.2(\mathrm{t})$. IR (neat) $\nu\left(\mathrm{cm}^{-1}\right) 3435,2930,1666,1651$, 1428, 1206, 932. HRMS (positive ESI) Calcd for $\mathrm{C}_{11} \mathrm{H}_{18} \mathrm{~N}_{2} \mathrm{O}_{3} \mathrm{Na}$ 
$\left[\mathrm{MNa}^{+}\right]$249.1210, found 249.1210. Following the procedure used to prepare 6, crude alcohol DCM $(140 \mathrm{~mL})$ was treated with Dess-Martin periodinane $(3.44 \mathrm{~g}, 8.11 \mathrm{mmol})$ for $14 \mathrm{~h}$. The usual workup (DCM) and purification (70\% EtOAc in hexanes) afforded a mixture of rotamers $44\left(1.44 \mathrm{~g}, 94 \%\right.$ over 2 steps) as a colorless oil. ${ }^{1} \mathrm{H}$ NMR $(300 \mathrm{MHz}$, $\left.\mathrm{CDCl}_{3}\right) \delta(\mathrm{ppm}) 9.68(\mathrm{~s}, 1 \mathrm{H}), 8.08(\mathrm{~s})$ and $(8.03)(\mathrm{s})(1 \mathrm{H}$, rotamers), $6.36(\mathrm{~d}, J=12.5 \mathrm{~Hz})$ and $6.34(\mathrm{~d}, J=12.5 \mathrm{~Hz})(1 \mathrm{H}$, rotamers $), 4.49-$ $4.17(\mathrm{~m}, 3 \mathrm{H}), 3.50(\mathrm{~s}, 3 \mathrm{H}), 3.41$ (dd. $2 \mathrm{H}, J=8.0,6.0 \mathrm{~Hz}), 2.50(\mathrm{~s}, 3 \mathrm{H})$, $1.88-1.78(\mathrm{~m}, 1 \mathrm{H}), 1.53-1.41(\mathrm{~m}, 1 \mathrm{H}) .{ }^{13} \mathrm{C} \mathrm{NMR}\left(75 \mathrm{MHz}, \mathrm{CDCl}_{3}\right) \delta$ (ppm) 201. Seven (d, rotamer), 202.2 (d), 162.4 (d), 162.0 (d, rotamer), $149.3(\mathrm{~d}), 149.1$ (d, rotamer), $114.9(\mathrm{~s}), 103.9$ (d, rotamer), $103.6(\mathrm{~d}), 56.4(\mathrm{q}), 56.3(\mathrm{q}$, rotamer $), 50.4(\mathrm{t}), 50.0(\mathrm{t}$, rotamer $), 45.5$ $(\mathrm{t}), 41.4(\mathrm{t}$, rotamer $), 36.0(\mathrm{t}$, rotamer $), 33.0(\mathrm{t}), 32.4(\mathrm{t}$, rotamer $), 30.9$ (d, rotamer), $30.3(\mathrm{~d}), 30.0(\mathrm{t})$. IR (neat) $\nu\left(\mathrm{cm}^{-1}\right)$ 2934, 2834, 1717, $1669,1652,1428,1396,1209,932$. HRMS (positive ESI) Calcd for $\mathrm{C}_{11} \mathrm{H}_{16} \mathrm{~N}_{2} \mathrm{O}_{3} \mathrm{Na}\left[\mathrm{MNa}^{+}\right]$247.1053, found 247.1055.

Methyl (E)-5-(2-(N-(Cyanomethyl)formamido)ethyl)-3-hydroxy-7-methoxy-2-methylenehept-6-enoate (45). Following the reported procedure, ${ }^{14} 44(374 \mathrm{mg}, 1.67 \mathrm{mmol})$ in 1,4-dioxane/ water $(1: 1)(8 \mathrm{~mL})$ was treated with methyl acrylate $(1.5 \mathrm{~mL}, 16.7$ $\mathrm{mmol}$ ) and DABCO (1.87 g, $16.7 \mathrm{mmol})$ for $72 \mathrm{~h}$. The usual workup (tert-Butyl methyl ether) and purification (70\% EtOAc in hexanes) afforded a mixture of rotamers and diastereomers $45(422 \mathrm{mg}, 82 \%)$ as a colorless oil. ${ }^{1} \mathrm{H}$ NMR $\left(300 \mathrm{MHz}, \mathrm{CDCl}_{3}\right) \delta(\mathrm{ppm}) 8.09(\mathrm{~s})$ and $8.08(\mathrm{~s})$ and $8.06(\mathrm{~s})$ and $8.04(\mathrm{~s})(1 \mathrm{H}), 6.39(\mathrm{~d}, J=12.5 \mathrm{~Hz})$ and $6.37(\mathrm{~d}, J=12.5$ $\mathrm{Hz})$ and $6.30(\mathrm{~d}, J=12.5 \mathrm{~Hz})$ and $6.28(\mathrm{~d}, J=12.5 \mathrm{~Hz})(1 \mathrm{H}), 6.23(\mathrm{~s})$ and $6.22(\mathrm{~s})(1 \mathrm{H}), 5.83(\mathrm{~s}) 5.81(\mathrm{~s})(1 \mathrm{H}), 4.51-4.39(\mathrm{~m}, 2 \mathrm{H}), 4.36-$ $4.17(\mathrm{~m}, 2 \mathrm{H}), 3.78(\mathrm{~s})$ and $3.77(\mathrm{~s})(3 \mathrm{H}), 3.55(\mathrm{~s})$ and $3.52(\mathrm{~s})(3 \mathrm{H})$, $3.45-3.40(\mathrm{~m}, 2 \mathrm{H}), 2.48(\mathrm{~d}, J=7.0 \mathrm{~Hz})$ and $2.78(\mathrm{~d}, J=7.0 \mathrm{~Hz})$ and 2.53-2.49 $(\mathrm{m})(1 \mathrm{H}), 2.34-2.22(\mathrm{~m})$ and $2.15-2.03(\mathrm{~m})(1 \mathrm{H}), 1.94-$ $1.42(\mathrm{~m}, 4 \mathrm{H}) .{ }^{13} \mathrm{C}$ NMR $\left(75 \mathrm{MHz}, \mathrm{CDCl}_{3}\right) \delta(\mathrm{ppm}) 167.0(\mathrm{~s}), 166.9$ (s), 162.6 (d), 162.5 (d), 162.1 (d), 162.0 (d), 149.3 (d), 149.2 (d), $148.9(\mathrm{~d}), 148.6(\mathrm{~d}), 148.4(\mathrm{~d}), 143.3(\mathrm{~s}), 143.2(\mathrm{~s}), 142.0(\mathrm{~s}), 125.5(\mathrm{t})$, $124.7(\mathrm{t}), 124.6(\mathrm{t}), 115.0(\mathrm{~s}), 114.9(\mathrm{~s}), 105.3(\mathrm{~d}), 105.2(\mathrm{~d}), 104.6(\mathrm{~d})$, 104.2 (d), 103.5 (d), 69.5 (d), 68.5 (d), $68.3(\mathrm{~d}), 56.4$ (q), $56.3(\mathrm{q}), 56.3$ $(\mathrm{q}), 56.2(\mathrm{q}), 56.1(\mathrm{q}), 52.0(\mathrm{q}), 52.0(\mathrm{q}), 51.9(\mathrm{q}), 46.0(\mathrm{t}), 45.8(\mathrm{t})$, $42.8(\mathrm{t}), 42.8(\mathrm{t}), 42.6(\mathrm{t}), 41.9(\mathrm{t}), 41.8(\mathrm{t}), 36.2(\mathrm{t}), 36.1(\mathrm{t}), 34.4(\mathrm{t})$, $33.4(\mathrm{~d}), 33.1(\mathrm{t}), 33.0(\mathrm{~d}), 32.8(\mathrm{t}), 32.7(\mathrm{~d}), 32.7(\mathrm{~d}), 32.0(\mathrm{t}), 30.2(\mathrm{t})$, $30.1(\mathrm{t}), 30.0(\mathrm{t})$. IR (neat) $\nu\left(\mathrm{cm}^{-1}\right) 3477,2950,1712,1668,1653$, 1434, 1397, 1207, 1142, 935. HRMS (positive ESI) Calcd for $\mathrm{C}_{15} \mathrm{H}_{22} \mathrm{~N}_{2} \mathrm{O}_{5} \mathrm{Na}\left[\mathrm{MNa}^{+}\right]$333.1421, found 333.1424.

Methyl (E)-3-Acetoxy-5-(2-( $N$-(cyanomethyl)formamido)ethyl)-7-methoxy-2-methylenehept-6-enoate (46). Following the reported procedure, ${ }^{20}$ a solution of DCC $(190 \mathrm{mg}, 0.92 \mathrm{mmol})$ and DMAP ( $8.7 \mathrm{mg}, 0.07 \mathrm{mmol})$ in DCM $(1 \mathrm{~mL})$ was added to a solution of $45(220 \mathrm{mg}, 0.71 \mathrm{mmol})$ and acetic acid $(43 \mu \mathrm{L}, 0.74 \mathrm{mmol})$ in DCM $(3 \mathrm{~mL})$. The mixture was stirred for $2 \mathrm{~h}$. Then, water was added. The usual workup (DCM) and purification (65\% EtOAc in hexanes) afforded a mixture of rotamers and diastereomers $46(136 \mathrm{mg}, 55 \%)$ as a colorless oil. ${ }^{1} \mathrm{H} \mathrm{NMR}\left(300 \mathrm{MHz}, \mathrm{CDCl}_{3}\right) \delta(\mathrm{ppm}) 8.09$ (s) and $8.08(\mathrm{~s})$ and $8.06(\mathrm{~s})$ and $8.05(\mathrm{~s})(1 \mathrm{H}), 6.29-6.22(\mathrm{~m}, 2 \mathrm{H}), 5.80(\mathrm{~s})$ and 5.75 $(\mathrm{s})(1 \mathrm{H}), 5.67(\mathrm{dd}, J=8.5,4.0 \mathrm{~Hz})$ and $5.59(\mathrm{dd}, J=8.5,4.0 \mathrm{~Hz})(1 \mathrm{H})$, $4.50-4.15(\mathrm{~m}, 3 \mathrm{H}), 3.78(\mathrm{~s})$ and $3.77(\mathrm{~s})(3 \mathrm{H}), 3.55(\mathrm{~s})$ and $3.52(\mathrm{~s})$ $(3 \mathrm{H}), 3.44-3.39(\mathrm{~m}, 2 \mathrm{H}), 2.08(\mathrm{~s})$ and $2.07(\mathrm{~s})(3 \mathrm{H}), 2.02-1.62(\mathrm{~m}$, $4 \mathrm{H}), 1.51-1.40(\mathrm{~m}, 1 \mathrm{H}) .{ }^{13} \mathrm{C}$ NMR $\left(75 \mathrm{MHz}, \mathrm{CDCl}_{3}\right) \delta(\mathrm{ppm}) 170.2$ (s), $169.8(\mathrm{~s}), 165.8$ (s), 165.7 (s), 162.4 (d), 161.8 (d), 149.5 (d), 149.1 (d), $148.7(\mathrm{~d}), 140.7(\mathrm{~s}), 140.5(\mathrm{~s}), 139.9(\mathrm{~s}), 139.7(\mathrm{~s}), 126.0(\mathrm{t}), 125.0$ (t), $124.9(\mathrm{t}), 114.9$ (s), $114.8(\mathrm{~s}), 104.9$ (d), 104.0 (d), 103.7 (d), 70.3 (d), $70.2(\mathrm{~d}), 70.1$ (d), 69.9 (d), $56.5(\mathrm{q}), 56.3(\mathrm{q}), 52.2(\mathrm{q}), 52.1$ (q), $46.0(\mathrm{t}), 45.6(\mathrm{t}), 42.3(\mathrm{t}), 41.4(\mathrm{t}), 41.3(\mathrm{t}), 36.3(\mathrm{t}), 34.2(\mathrm{t}), 33.9(\mathrm{~d})$, $33.8(\mathrm{~d}), 33.2(\mathrm{~d}), 33.1(\mathrm{~d}), 32.8(\mathrm{t}), 30.3(\mathrm{t}), 30.1(\mathrm{t}), 21.2(\mathrm{q}), 21.1(\mathrm{q})$. IR (neat) $\nu\left(\mathrm{cm}^{-1}\right) 2935,1736,1720,1675,1653,1434,1370,1232,935$. HRMS (positive ESI) Calcd for $\mathrm{C}_{17} \mathrm{H}_{24} \mathrm{~N}_{2} \mathrm{O}_{6} \mathrm{Na}\left[\mathrm{MNa}^{+}\right]$375.1527, found 375.1531 .

Methyl (E)-4-Acetoxy-2-cyano-7-(methoxymethylene)octahydro-1,6-ethanoindole-3a-carboxylate (51). $\mathrm{Tf}_{2} \mathrm{O}(53 \mu \mathrm{L}$, $0.31 \mathrm{mmol})$ was added to a solution of $46(100 \mathrm{mg}, 0.28 \mathrm{mmol})$ and DTBMP $(64.1 \mathrm{mg}, 0.31 \mathrm{mmol})$ in DCM $(3.0 \mathrm{~mL})$ at rt. After $5 \mathrm{~min}$, the mixture was diluted with chlorobenzene $(500 \mathrm{~mL})$ and heated to 125 ${ }^{\circ} \mathrm{C}$. Then $i$ - $\mathrm{Pr}_{2} \mathrm{NEt}$ (494 $\mu \mathrm{L}, 2.84 \mathrm{mmol}$ ) was added. The mixture was stirred for $20 \mathrm{~min}$. Saturated aqueous $\mathrm{NaHCO}_{3}$ was added. The usual workup (DCM) and purification (30 to 60\% EtOAc in hexanes) afforded two separable portions of two diastereomers of 51: portion 1 $(27.8 \mathrm{mg})$ as a mixture of a colorless oil and solid and portion 2 (17.1 $\mathrm{mg}$ ) as a mixture of a yellow oil and solid (47\% global yield). Portion 1 (two diastereomers): ${ }^{1} \mathrm{H}$ NMR $\left(300 \mathrm{MHz}, \mathrm{CDCl}_{3}\right) \delta(\mathrm{ppm}) 6.10(\mathrm{~s})$, and $6.00(\mathrm{~s})$, and $5.99(\mathrm{~s})(1 \mathrm{H}), 5.38(\mathrm{dd}, J=12.0,7.0 \mathrm{~Hz})$, and $6.20(\mathrm{dd}$, $J=11.0,7.0 \mathrm{~Hz})(1 \mathrm{H}), 4.47(\mathrm{~s})$, and $4.46(\mathrm{~s})$, and $4.20(\mathrm{~s})(1 \mathrm{H}), 3.97$ (ddd, $J=10.0,7.0,2.5 \mathrm{~Hz})$, and $3.90(\mathrm{t}, J=8.5 \mathrm{~Hz})(1 \mathrm{H}), 3.71(\mathrm{~s})$, and $3.70(\mathrm{~s})$, and $3.69(\mathrm{~s})$, and $3.68(\mathrm{~s})(3 \mathrm{H}), 3.64(\mathrm{~s})$, and $3.58(\mathrm{~s})$, and 3.55 (s) $(3 \mathrm{H}), 3.31-3.29(\mathrm{~m}, 1 \mathrm{H}), 3.27-3.09(\mathrm{~m}, 1 \mathrm{H}), 2.62-2.42(\mathrm{~m}, 3 \mathrm{H})$, 2.31 (ddd, $1 \mathrm{H}, J=14.0,11.0,7.0 \mathrm{~Hz}), 2.03(\mathrm{~s})$, and $2.00(\mathrm{~s})(3 \mathrm{H}), 1.98-$ $1.87(\mathrm{~m}, 1 \mathrm{H}), 1.81-1.75(\mathrm{~m}, 1 \mathrm{H}), 1.36(\mathrm{ddd}, 1 \mathrm{H}, J=14.0,12.0,3.5 \mathrm{~Hz})$. ${ }^{13} \mathrm{C} \mathrm{NMR}\left(75 \mathrm{MHz}, \mathrm{CDCl}_{3}\right) \delta 174.5(\mathrm{~s}), 170.8(\mathrm{~s}), 170.3(\mathrm{~s}), 170.0(\mathrm{~s})$, $143.4(\mathrm{~d}), 143.2(\mathrm{~d}), 141.9$ (d), $120.8(\mathrm{~s}), 117.6(\mathrm{~s}), 110.6(\mathrm{~s}), 110.4(\mathrm{~s})$, $109.8(\mathrm{~s}), 71.0,69.8,65.5,62.5,60.1,60.0,59.4(\mathrm{~s}), 58.1(\mathrm{~s}), 57.4(\mathrm{~s})$, $54.4,52.9,52.0,51.7,45.7(\mathrm{t}), 45.4(\mathrm{t}), 42.2(\mathrm{t}), 38.4(\mathrm{t}), 38.0(\mathrm{t}), 36.6$ $(\mathrm{t}), 34.7(\mathrm{t}), 33.0(\mathrm{t}), 32.5(\mathrm{t}), 29.8(\mathrm{t}), 27.6(\mathrm{~d}), 27.3(\mathrm{t}), 26.9(\mathrm{t}), 26.2$ $(\mathrm{t}), 25.1(\mathrm{~s}), 21.3(\mathrm{q}), 21.20(\mathrm{q})$. IR (neat) $\nu\left(\mathrm{cm}^{-1}\right) 2950,2935,1730$, 1694, 1434, 1373, 1235, 1203, 1127. HRMS (positive ESI) Calcd for $\mathrm{C}_{17} \mathrm{H}_{22} \mathrm{~N}_{2} \mathrm{O}_{5} \mathrm{Na}\left[\mathrm{MNa}^{+}\right]$357.1421, found 357.1435. Portion 2 (two diastereomers): $\left.{ }^{1} \mathrm{H} \mathrm{NMR} \mathrm{(300} \mathrm{MHz}, \mathrm{CDCl}_{3}\right) \delta(\mathrm{ppm}) 6.12(\mathrm{~s})$, and $6.09(\mathrm{~s})$, and $6.07(\mathrm{~s})$ and $6.02(\mathrm{~s})$, and $5.96(\mathrm{~s})(1 \mathrm{H}), 5.47(\mathrm{dd}, J=10.5$, $7.0 \mathrm{~Hz})$, and $5.32(\mathrm{dd}, J=11.5,7.0 \mathrm{~Hz})(1 \mathrm{H}), 4.04-3.93(\mathrm{~m}, 1 \mathrm{H}), 3.86$ $(\mathrm{dd}, 1 \mathrm{H}, J=11.0,6.0 \mathrm{~Hz}), 3.74(\mathrm{~s})$, and $3.72(\mathrm{~s})$, and $3.69(\mathrm{~s})(3 \mathrm{H}), 3.63$ $(\mathrm{s})$, and $3.61(\mathrm{~s})$, and $3.58(\mathrm{~s})(3 \mathrm{H}), 3.31-3.13(\mathrm{~m}, 1 \mathrm{H}), 3.07-2.88(\mathrm{~m}$, $1 \mathrm{H}), 2.81-2.69(\mathrm{~m}, 1 \mathrm{H}), 2.64-2.41(\mathrm{~m}, 2 \mathrm{H}), 2.26(\mathrm{ddd}, 1 \mathrm{H}, J=14.0$, $11.5,7.0 \mathrm{~Hz}), 1.99(\mathrm{~s}, 3 \mathrm{H}), 1.78-1.66(\mathrm{~m}, 1 \mathrm{H}), 1.39-1.12(\mathrm{~m}, 1 \mathrm{H}) .{ }^{13} \mathrm{C}$ NMR $\left(75 \mathrm{MHz}, \mathrm{CDCl}_{3}\right) \delta 174.6(\mathrm{~s}), 171.5(\mathrm{~s}), 171.0(\mathrm{~s}), 170.3(\mathrm{~s})$, $170.2(\mathrm{~s}), 169.9(\mathrm{~s}), 142.3(\mathrm{~d}), 141.7(\mathrm{~d}), 141.6(\mathrm{~d}), 118.0(\mathrm{~s}), 117.9(\mathrm{~s})$, $117.5(\mathrm{~s}), 111.5(\mathrm{~s}), 111.2(\mathrm{~s}), 110.1(\mathrm{~s}), 109.5(\mathrm{~s}), 74.8(\mathrm{~d}), 73.8(\mathrm{~d})$, $71.5(\mathrm{~d}), 71.0(\mathrm{~d}), 68.2(\mathrm{~d}), 66.6(\mathrm{~d}), 61.5(\mathrm{q}), 61.2(\mathrm{q}), 60.5(\mathrm{q}), 60.1$ (q), $60.0(\mathrm{q}), 59.9(\mathrm{q}), 58.6(\mathrm{~s}), 58.3(\mathrm{~s}), 58.1(\mathrm{~s}), 55.7,54.1,53.0,52.9$, 52.4, 52.2, $46.3(\mathrm{t}), 45.3,44.7(\mathrm{t}), 42.4(\mathrm{t}), 40.4(\mathrm{t}), 39.5(\mathrm{t}), 36.1(\mathrm{t})$, $33.3(\mathrm{~s}), 33.1(\mathrm{t}), 31.3(\mathrm{t}), 31.2(\mathrm{t}), 31.1(\mathrm{t}), 30.5,29.9(\mathrm{~s}), 29.8(\mathrm{~s}), 26.3$ $(\mathrm{t}), 25.0(\mathrm{~s}), 23.1(\mathrm{q}), 23.0(\mathrm{q}), 21.2(\mathrm{q}), 21.2(\mathrm{q}) . \operatorname{IR}($ neat $) \nu\left(\mathrm{cm}^{-1}\right)$ 2948, 2933, 1730, 1693, 1435, 1372, 1236, 1206, 1127. HRMS (positive ESI) Calcd for $\mathrm{C}_{17} \mathrm{H}_{22} \mathrm{~N}_{2} \mathrm{O}_{5} \mathrm{Na}\left[\mathrm{MNa}^{+}\right]$357.1421, found 357.1392.

\section{ASSOCIATED CONTENT}

\section{Supporting Information}

The Supporting Information is available free of charge on the ACS Publications website at DOI: 10.1021/acs.joc.7b00345.

Copies ${ }^{1} \mathrm{H}$ and ${ }^{13} \mathrm{C}$ NMR spectra for all new compounds, and ORTEP representation of compound 51 (PDF) Crystallographic data (CIF)

\section{AUTHOR INFORMATION}

\section{Corresponding Author}

*E-mail: Guillaume.Belanger@USherbrooke.ca.

ORCID $\odot$

Guillaume Bélanger: 0000-0002-7454-8760

Notes

The authors declare no competing financial interest.

\section{ACKNOWLEDGMENTS}

This research was supported by the Natural Science and Engineering Research Council (NSERC) of Canada and the Université de Sherbrooke.

\section{REFERENCES}

(1) For racemic syntheses of condylocarpine, isocondylocarpine, lagunamine, and condyfoline, see: (a) Kuehne, M. E.; Brook, C. S.; Frasier, D. A.; Xu, F. J. Org. Chem. 1995, 60, 1864. (b) Ban, Y.; Yoshida, K.; Goto, J.; Oishi, T.; Takeda, E. Tetrahedron 1983, 39, 3657. (c) Amat, 
M.; Hadida, S.; Pshenichnyi, G.; Bosch, J. J. Org. Chem. 1997, 62, 3158. For enantioselective syntheses of condylocarpine, isocondylocarpine, tubotaiwine, and condyfoline, see: (d) Martin, C. L.; Nakamura, S.; Otte, R.; Overman, L. E. Org. Lett. 2011, 13, 138. (e) Amat, M.; Coll, M. D.; Bosch, J. Tetrahedron: Asymmetry 1997, 8, 935. For a selection of recent articles on the synthesis of or approach towards Apidospermatan-type alkaloids, see: (f) Zhao, S.; Andrade, R. B. J. Org. Chem. 2017, 82, 521. (g) Maertens, G.; Canesi, S. Chem. - Eur. J. 2016, 22, 7090. (h) Ding, M.; Tong, X.; Li, D.; Liang, K.; Zhou, A.; Zuo, Z.; Xia, C. Tetrahedron Lett. 2015, 56, 5460. (i) Beemelmanns, C.; Gross, S.; Reissig, H.-U. Chem. Eur. J. 2013, 19, 17801. (j) Cannon, J. S.; Overman, L. E. Angew. Chem., Int. Ed. 2012, 51, 4288. (k) Martin, D. B. C.; Vanderwal, C. D. Chem. Sci. 2011, 2, 649. (1) Jones, S. B.; Simmons, B.; Mastracchio, A.; MacMillan, D. W. C. Nature 2011, 475, 183. (m) Sirasani, G.; Paul, T.; Dougherty, W., Jr.; Kassel, S.; Andrade, R. B. J. Org. Chem. 2010, 75, 3529.

(2) For sequential Vilsmeier-Haack cyclization and intramolecular azomethine ylide cycloaddition, see: (a) Lévesque, F.; Bélanger, G. Org. Lett. 2008, 10, 4939. (b) Bélanger, G.; Darsigny, V.; Doré, M.; Lévesque, F. Org. Lett. 2010, 12, 1396. (c) Bélanger, G.; Boudreault, J.; Lévesque, F. Org. Lett. 2011, 13, 6204. (d) Bélanger, G.; Lévesque, F.; Boudreault, J. J. Org. Chem. 2016, 81, 9247.

(3) Fischer indolization on compounds such as $\mathbf{4}$ is known to fail in aspidospermatan-type alkaloids synthesis. See: Kuehne, M. E.; Frasier, D. A.; Spitzer, T. D. J. Org. Chem. 1991, 56, 2696.

(4) For a related transformation, see: Smith, M. W.; Snyder, S. A. J. Am. Chem. Soc. 2013, 135, 12964.

(5) For a related transformation, see: Moustafa, G. A. I.; Saku, Y.; Aoyama, H.; Yoshimitsu, T. Chem. Commun. 2014, 50, 15706.

(6) Allegretti, P. A.; Ferreira, E. M. J. Am. Chem. Soc. 2013, 135, 17266.

(7) The asymmetric version of this reaction was attempted, but unusable enantioselectivities were obtained. The same results were obtained when substrates with protecting groups other than TBS were tested. At this point, we decided to proceed with the racemic compound, although chiral induction will eventually need to be addressed before completing the syntheses of targeted natural products. Chi, Y.; Guo, L.; Kopf, N. A.; Gellman, S. H. J. Am. Chem. Soc. 2008, 130, 5608.

(8) Tsuji, Y.; Funato, M.; Ozawa, M.; Ogiyama, H.; Kajita, S.; Kawamura, T. J. Org. Chem. 1996, 61, 5779.

(9) Diastereomers were separated, but relative stereochemistry could not be assigned. The sequence was independently continued with both diastereomers that ultimately led to the same key step substrate 6 .

(10) Battersby, A. R.; Baker, M. G.; Broadbent, H. A.; Fookes, C. J. R.; Leeper, F. J. J. Chem. Soc., Perkin Trans. 1 1987, 2027.

(11) (a) Katritzky, A. R.; Chang, H.-X.; Yang, B. Synthesis 1995, 1995, 503. (b) Pasqua, A. E.; Matheson, M.; Sewell, A. L.; Marquez, R. Org. Process Res. Dev. 2011, 15, 467.

(12) TBAT induced polymerization of $\mathrm{N}$-phenylmaleimide. This could account for the poor results.

(13) Azomethine ylides are known to react faster with electrondeficient dipolarophiles. See: Coldham, I.; Watson, L.; Adams, H.; Martin, N. G. J. Org. Chem. 2011, 76, 2360. See also ref 2d.

(14) Yu, C.; Liu, B.; Hu, L. J. Org. Chem. 2001, 66, 5413.

(15) Compound after Baylis-Hillman reaction was obtained as a mixture of amide rotamers. The relative stereochemistry of the diastereomer obtained could not be assigned at this stage, but was deduced from the relative stereochemistry observed on the X-ray structure of cycloadduct $\mathbf{5 1}$.

(16) A TES group was chosen for compatibility with the key step, and the greater yields for TES protection were compared with $\mathrm{Bn}$ protection (cf. protection of 30). In the case of compound 30, TES protection was not possible because the generation of azomethine ylide from iminium ion 35 required desilylation conditions that would have cleaved the protecting group.

(17) The greater stability of the unsaturated ester as the dipolarophile (compared to enone 6) allowed the Vilsmeier-Haack reaction to proceed at rt.

(18) Deprotonation of iminium ion 39 at lower $\left(40{ }^{\circ} \mathrm{C}\right.$ in DCE) or higher temperatures $\left(120^{\circ} \mathrm{C}\right.$ in chlorobenzene) did not lead to improved yields, except that desilylation of OTES occurred under the latter conditions. The corresponding alcohol formed is shown in the Supporting Information (SI). Switching the TES to an acetate as the protecting group on 33 led to the corresponding cycloadduct but still in traces, regardless of the cycloaddition temperature. Other bases of different strengths were also tested (DIPEA, DABCO, AcONa, $\mathrm{Cs}_{2} \mathrm{CO}_{3}$ ). Unfortunately, we were not able to influence the outcome of the reaction, and $\mathbf{4 1}$ was always the sole isolated product.

(19) The isolated quantity of pure $Z$-isomer of $\mathbf{4 2}$ was not sufficient to complete the sequence up to the key cyclization step.

(20) Compound $\mathbf{4 5}$ proved to be very sensitive to either basic or acidic conditions. Hence, TES protection (TESCl, base) or benzylation $(\mathrm{BnBr}$, base or benzyl trichloroacetimidate, $\mathrm{TsOH}$ ) resulted in poor yields. Only acetylation under neutral esterification conditions was satisfactory. Hassner, A.; Alexanian, V. Tetrahedron Lett. 1978, 19, 4475.

(21) Only traces of cycloadduct were obtained upon heating to $80^{\circ} \mathrm{C}$, a temperature that was not sufficient to promote cycloaddition.

(22) An ORTEP representation could be found in the SI. Crystallographic data for this compound have been deposited with the Cambridge Crystallographic Data Centre (CCDC no. 1542684). Coordinates can be obtained at http://www.ccdc.cam.ac.uk/deposit.

(23) For representation of X-ray structure: CYLview, 1.0b; Legault, C. Y. Université de Sherbrooke, 2009 (http://www.cylview.org).

(24) Anderson, A. G.; Stang, P. J. Org. Synth. 1981, 60, 34. 